\title{
Quasi- $L^{p}$ norm orthogonal Galerkin expansions in sums of Jacobi polynomials
}

\section{Orthogonal expansions}

\author{
Philip W. Livermore • Glenn R. Ierley
}

Received: 16 March 2009 / Accepted: 16 November 2009 /

Published online: 27 November 2009

(C) The Author(s) 2009. This article is published with open access at Springerlink.com

\begin{abstract}
In the study of differential equations on [-1,1] subject to linear homogeneous boundary conditions of finite order, it is often expedient to represent the solution in a Galerkin expansion, that is, as a sum of basis functions, each of which satisfies the given boundary conditions. In order that the functions be maximally distinct, one can use the Gram-Schmidt method to generate a set orthogonal with respect to a particular weight function. Here we consider all such sets associated with the Jacobi weight function, $w(x)=$ $(1-x)^{\alpha}(1+x)^{\beta}$. However, this procedure is not only cumbersome for sets of large degree, but does not provide any intrinsic means to characterize the functions that result. We show here that each basis function can be written as the sum of a small number of Jacobi polynomials, whose coefficients are found by imposing the boundary conditions and orthogonality to the first few basis functions only. That orthogonality of the entire set follows-a property we term "auto-orthogonality"-is remarkable. Additionally, these basis functions are shown to behave asymptotically like individual Jacobi polynomials and share many of the latter's useful properties. Of particular note is that these basis sets retain the exponential convergence characteristic of Jacobi expansions for expansion of an arbitrary function satisfying the boundary conditions imposed. Further, the associated error is asymptotically minimized in an $L^{p(\alpha)}$ norm given the appropriate choice of $\alpha=\beta$. The rich algebraic structure
\end{abstract}

\footnotetext{
P. W. Livermore · G. R. Ierley

Institute of Geophysics and Planetary Physics, Scripps Institution of Oceanography, University of California, San Diego, La Jolla, CA 92093-0225, USA

e-mail: p.w.livermore@leeds.ac.uk

Present Address:

P. W. Livermore $(\bowtie)$

School of Earth and Environment, University of Leeds, Leeds, LS2 9JT, United Kingdom e-mail: phil.livermore@googlemail.com
} 
underlying these properties remains partially obscured by the rather difficult form of the non-standard weighted integrals of Jacobi polynomials upon which our analysis rests. Nevertheless, we are able to prove most of these results in specific cases and certain of the results in the general case. However a proof that such expansions can satisfy linear boundary conditions of arbitrary order and form appears extremely difficult.

Keywords Orthogonal polynomial - Galerkin expansion - Jacobi polynomial • Spectral method. Exponential convergence

\section{Introduction}

Spectral methods are an extremely efficient way of solving many numerical problems since, if the function for which one is solving is smooth, only a few terms are typically required to represent it to high accuracy. This efficiency rests on the spectral coefficients, $f_{n}$, tending to zero faster than any algebraic power of $n$. Orthogonal polynomials (see Ismail [9] for a thorough review) offer an attractive and widely used framework on which to build such methods. One such class, the Jacobi polynomials, converges at an exponential rate on the interval $[-1,1]$, of which particular well-known examples are Chebyshev polynomials of the first and second kinds and Legendre polynomials. These latter families have the additional property of asymptotically minimizing the error between an arbitrary function and its projection as measured in the $L^{\infty}$, $L^{1}$ and $L^{2}$ norms respectively [15]. For the Chebyshev polynomials of the first and second kinds, these properties are manifested respectively by equalripple, i.e. they oscillate uniformly, and equal-area, i.e. the area under the curve between any two consecutive zeros is constant.

It is often the case that certain linear homogeneous boundary conditions must be satisfied by the unknown solution. ${ }^{1}$ In this event, it is often expedient to use an expansion in spectral functions that individually satisfy these conditions explicitly, for then their sum must also; we then need solve only for the spectral coefficients in ensuing analysis, effectively disregarding the boundary conditions from that point on. An arbitrary sum of Jacobi polynomials satisfies no particular condition at $x= \pm 1$; nonetheless, a tailor-made basis set can be fashioned by taking particular linear combinations of these polynomials [3]. However, the property of orthogonality is in general immediately lost and there is no guarantee that the resulting set of functions, although formally useful, is numerically well-conditioned. This is easily illustrated by combining Chebyshev polynomials of the first kind, $T_{n}(x)$, of various degrees to fit the

\footnotetext{
${ }^{1}$ If the given boundary conditions are not homogeneous they can always be made so with the addition of an appropriate function to the unknown solution, with the associated modification of the differential equation.
} 
boundary condition $f(1)+f^{\prime}(1)=0$. Two possible sets of basis functions are $\left\{\psi_{n}^{(1)}\right\}$ and $\left\{\psi_{n}^{(2)}\right\}$, each formally spanning the same function space, where

$$
\begin{aligned}
& \psi_{n}^{(1)}(x)=T_{n}(x)-\left(n^{2}+1\right) T_{0}(x), \\
& \psi_{n}^{(2)}(x)=\left((n-1)^{2}+1\right) T_{n}(x)-\left(n^{2}+1\right) T_{n-1}(x) .
\end{aligned}
$$

We note that $\psi_{n}^{(1)}$ becomes increasingly ill-conditioned as $n$ increases, as when normalized, $\psi_{n}^{(1)}(x) \sim T_{0}$, which is independent of $n$. The second case, $\psi_{n}^{(2)}$, is better conditioned but is neither an orthogonal set, nor close to equalripple. An alternative approach is to use a Gram-Schmidt procedure [5] with the boundary condition to generate an orthogonal set, by writing $\psi_{n}^{(3)}=$ $\sum_{i=0}^{n} a_{n} P_{n}^{(\alpha, \beta)}(x)$, where the $a_{i}$ are determined from imposing orthogonality (with weight function $\left.w(x)=(1-x)^{\alpha}(1+x)^{\beta}\right)$ to $\psi_{i}^{(3)}, i=1, \ldots, n-1$, the boundary condition and some normalization condition. ${ }^{2}$ Although guaranteed to produce an orthogonal set, this algorithm is cumbersome for large $n$ and provides no means to prove any asymptotic, or other, properties of the functions.

In this paper, we show that a drastically truncated Gram-Schmidt method suffices for constructing orthogonal basis sets on $[-1,1]$ that satisfy any number of linear boundary conditions at $x= \pm 1$. For the particular case of a single first-order boundary condition at $x=1$, we will show that the following representation generates an orthogonal set:

$$
\Psi_{n}(x)=\sum_{i=1}^{3} c_{i} P_{n-i+1}^{(\alpha+2, \beta)}(x), \quad n \geq 2
$$

where $\Psi_{1}(x)$ is the unique polynomial of degree one satisfying the boundary condition. The three values of $c_{i}$ are determined by imposing the single condition of orthogonality to $\Psi_{1}(x)$, the boundary condition, and a normalization. It is far from evident that such a procedure will yield the same set of functions as the full Gram-Schmidt process, which explicitly enforces mutual orthogonality; yet it does. Additionally, as we later indicate, $\Psi_{n}(x) \sim P_{n}^{(\alpha, \beta)}$ as $n \rightarrow \infty$ and the expansion coefficients converge exponentially whenever those of the associated expansion in $P_{n}^{(\alpha, \beta)}$ do so. Hence by choosing $\alpha=\beta=-1 / 2, \alpha=\beta=1 / 2$, or $\alpha=\beta=0$, we thus determine basis sets that are asymptotically similar to the Chebyshev polynomials of the first or second kinds, or the Legendre polynomials, respectively and the asymptotic error between a given function and its truncated Galerkin expansion is consequently minimized in the $L^{\infty}$, $L^{1}$, or $L^{2}$ norms. The provenance of this result could only be obscured by brute-force application of the usual Gram-Schmidt algorithm.

We begin with a simple motivational example that illustrates some of the key issues presented subsequently in more detail. Suppose that we wish

\footnotetext{
${ }^{2}$ Using the natural polynomials $x^{n}$ in place of Jacobi polynomials is formally equivalent but leads to severe numerical problems as the $a_{n}$ grow exponentially with $n$ and accuracy is rapidly lost in finite precision.
} 
to construct a basis set capable of representing a function on $[-1,1]$ that satisfies the homogeneous boundary condition $f(1)=0$, and for which all members are mutually orthogonal with respect to the weight function $w(x)=$ $(1-x)^{\alpha}(1+x)^{\beta}$. This may be written as

$$
\Psi_{n}(x)=(1-x) P_{n-1}^{(\alpha+2, \beta)}(x), \quad n \geq 1
$$

since $\Psi_{n}(1)$ clearly vanishes and applying the standard orthogonality relation of Jacobi polynomials we see that

$$
\int \Psi_{n}(x) \Psi_{m}(x) w(x) d x=\int_{-1}^{1} P_{n-1}^{(\alpha+2, \beta)} P_{m-1}^{(\alpha+2, \beta)}(1-x)^{2+\alpha}(1+x)^{\beta} d x=h_{n} \delta_{n m}
$$

for some constants $h_{n}$.

Using the standard index recurrence relations (64) and (66), we can write

$$
\Psi_{n}(x)=c_{1}(n) P_{n}^{(\alpha+2, \beta)}+c_{2}(n) P_{n-1}^{(\alpha+2, \beta)}+c_{3}(n) P_{n-2}^{(\alpha+2, \beta)}, \quad n \geq 2
$$

for coefficients $c_{i}(n)$, which take the (unnormalized) form

$$
\begin{aligned}
& c_{1}(n)=n(\beta+\alpha+2 n)(\alpha+\beta+n+2), \\
& c_{2}(n)=-(\beta+1+2 n+\alpha)\left(2 n^{2}+2 n \beta+2 n+2 n \alpha+\beta \alpha+\alpha^{2}+3 \alpha+2+\beta\right), \\
& c_{3}(n)=(\alpha+n+1)(\beta+n-1)(\beta+\alpha+2 n+2),
\end{aligned}
$$

for $n \geq 2$ and $\Psi_{1}(x)=1-x$. Note that the $c_{i}$ take on the ratio $[1,-2,1]$ as $n \rightarrow \infty$, a property that has great significance since the same asymptotic behavior arises from writing $P_{n}^{(\alpha, \beta)}(x)$ in the form of (2) by applying (66) twice. It follows that $\Psi_{n}(x) \sim P_{n}^{(\alpha, \beta)}(x)$ for large $n$ (except possibly in boundary layers).

Suppose now we consider constructing a second basis set that satisfies $f(1)=0$ of the form (2) in a truncated-Gram-Schmidt method, where the three coefficients $c_{i}$ are found by imposing the boundary condition, some normalization condition (which for the moment we ignore) and orthogonality only to the first polynomial element $(1-x)$. It is clear that such a scheme must reconstruct the mutually orthogonal basis set that we first thought of; however, in contrast with a typical Gram-Schmidt construction, we only impose orthogonality with respect to one polynomial rather than all the polynomials of lesser degree.

We now extend this simple case to one where we cannot write down the explicit form of the basis as in (1), by considering the single boundary condition $f^{\prime}(1)=0$. We attempt to find an unnormalized basis set of the form (2) for $n \geq 2$ by using only the boundary condition and orthogonality with respect to the first element of the basis set. The lowest degree function is the unique polynomial of degree one that satisfies the boundary condition: $\Psi_{1}(x)=1$.

Using the boundary condition relation (69) we find that the three coefficients $c_{i}(n)$ are polynomials of degree five, which we do not list here for brevity, but are given as a special case of the more general boundary condition $\mu f^{\prime}(1)+(1-\mu) f(1)$ in Appendix B. In fact the $c_{i}$ are in the ratio of $[1,-2,1]$ as $n \rightarrow \infty$, thus again $\Psi_{n}(x) \sim P_{n}^{(\alpha, \beta)}(x)$ for any value of $\mu$ and in this sense 
the basis functions become asymptotically independent of particular boundary conditions. It is far from apparent that the $\Psi_{n}$ resulting from this construction should prove to be mutually orthogonal, since we have only imposed orthogonality to $\Psi_{1}(x)$. We term this remarkable property 'auto-orthogonality'. Although one can prove the property after the fact by integrating the product of basis functions $\Psi_{n}$ and $\Psi_{m}$, this fails to do more than simply to confirm orthogonality. A more illuminating method is taken up in Section 4 where we extend this construction to boundary conditions of any order at $x= \pm 1$.

Before embarking on anything further, however, we provide the reader with some plots that illustrate the structure of the generated functions. Figure 1 shows $\Psi_{n}$ for $n=5,10,15$ for two sets of boundary conditions: $\Psi_{n}(-1)=$ $\Psi_{n}^{\prime}(1)=0$ in (a) and $\Psi_{n}^{\prime \prime}(1)=\Psi_{n}(1)=0$ in (b). The distinction between these examples, that the boundary conditions are imposed on two sides rather than just one, is important only in what we are able to prove in later sections; it is apparent that the algorithm works in all cases. To illustrate the asymptotic behavior as $n \rightarrow \infty$, the functions in (a) have $\alpha=\beta=-1 / 2$, whilst in (b) $\alpha=\beta=1 / 2$. It is striking how closely the equal-ripple result is achieved in (a) and similarly the equal-area property in (b).

The remainder of the paper is arranged as follows. In the next section, we provide a construction that defines an auto-orthogonal set for any linear boundary conditions involving derivatives of finite degree. The construction simplifies considerably in the case that the boundary conditions are imposed only on one side (that is, at $x=1$ or $x=-1$, but not both). In subsequent sections, we lay out various results and machinery that we use to prove autoorthogonality in specific cases. The starting point, supplied in Section 3, is an appropriate analytic expression for the weighted integral of products of Jacobi polynomials. Given a specific set of boundary conditions, one can simply

(a)

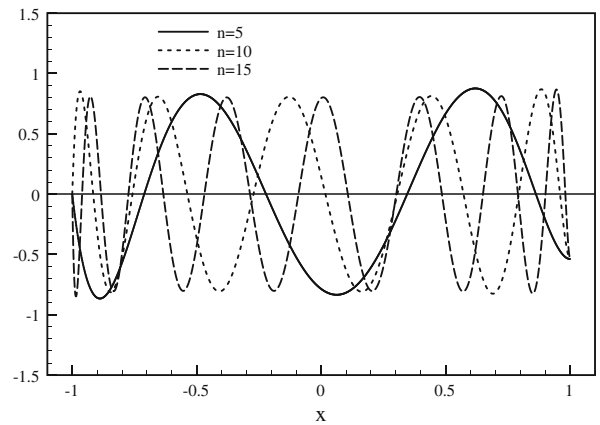

(b)

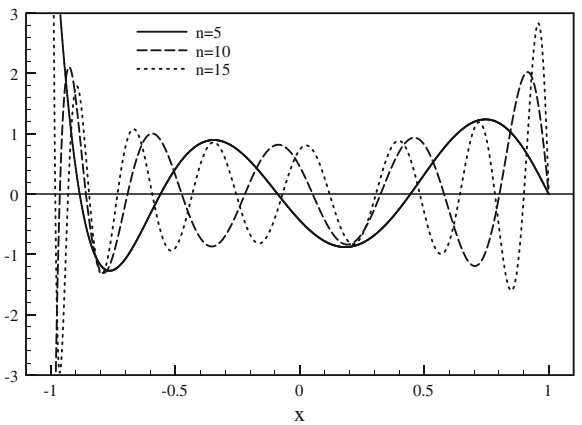

Fig. 1 Plots of basis functions for $n=5,10,15$. In $\mathbf{a}, \alpha=\beta=-1 / 2$ and the functions satisfy the two-sided boundary conditions $\Psi_{n}(-1)=\Psi_{n}^{\prime}(1)=0$; in $\mathbf{b}, \alpha=\beta=1 / 2$ and the functions satisfy the one-sided boundary condition $\Psi_{n}^{\prime \prime}(1)=\Psi_{n}(1)=0$. Note the quasi-equal-area property of the functions in $\mathbf{b}$ and the quasi-equal-ripple property in $\mathbf{a}$. The functions are normalized by relation (26). In this normalization $\Psi_{n}(1)$ tends to a constant only for $n \rightarrow \infty$. In a the transient is simply too small to be seen for the values of $n$ plotted 
accept the construction we give, compute the basis set and, as commented above, prove mutual orthogonality a posteriori. A more insightful approach in Section 4 is to investigate the space of auto-orthogonal polynomials and show that it contains sufficient degrees of freedom to satisfy boundary conditions up to a given order. This attack relies on the fact that the matrix whose elements represent the inner product of pairs of basis functions can be written as a sum of special outer products, whose form we give. In simple cases we are able to prove auto-orthogonality, in particular, for arbitrary second-order (one-sided) boundary conditions. Additionally, we suggest a possible approach to the general case. In Section 5 we indicate the asymptotic character of the basis sets and lastly we provide an extension of the construction in Section 6.1. We conclude with a summary of our findings and discuss the immediate extensions of this study to numerical schemes in spherical and cylindrical geometries. In an accompanying article [11] we provide further numerical details related to the implementation of the Galerkin basis sets; a listing of basis functions satisfying a comprehensive set of physically motivated boundary conditions can be found at [12].

\section{Construction of an auto-orthogonal basis set for arbitrary boundary conditions}

\subsection{One-sided boundary conditions}

As motivated above, we consider a set of $M$ boundary conditions involving derivatives of the function at $x=1$ only and of degree at most $N-1$. The imposition of boundary conditions at $x=-1$ will be considered shortly. Note that $M$ cannot exceed $N$ or the problem is overspecified (unless the conditions are degenerate) and there is no solution. An appropriate representation involves a sum of $N+1$ Jacobi polynomials of neighboring degree, whose coefficients are determined by the $M$ boundary conditions, one normalization condition, (which we ignore) and $N-M$ orthogonality conditions. Thus

$$
\Psi_{n}(x)=\sum_{i=1}^{N+1} c_{i}(n) P_{M+n-i}^{(\alpha+N, \beta)}(x), \quad n \geq N-M+1 .
$$

For $n \leq N-M$, the above form breaks down (as the degree of the constituent Jacobi polynomials must be non-negative) and the first few basis functions must instead be found by using the standard Gram-Schmidt process, by determining the coefficients $c_{i}$ in

$$
\Psi_{n}(x)=\sum_{i=1}^{M+n} c_{i}(n) P_{i-1}^{(\alpha+N, \beta)}(x), \quad n \leq N-M
$$

and imposing the $M$ boundary conditions, one normalization condition, and orthogonality to $\Psi_{m}$ with weight $w(x)$ for $1 \leq m<n$. One example of this is the 
basis set discussed in the introduction, satisfying $\mu f^{\prime}(1)+(1-\mu) f(1)$, which is of the above form with $N=2, M=1$. It is also noteworthy that increasing $M$ does not change the essential structure of the basis, but merely increases the degree of all the polynomials; this is caused by providing each $\Psi_{n}$ with the degrees of freedom it needs to satisfy the extra boundary conditions. The functions $\Psi_{n}$ have the property that

$$
\int_{-1}^{1} \Psi_{n}(x) \Psi_{m}(x)(1-x)^{\alpha}(1+x)^{\beta} d x=k_{n} \delta_{n m},
$$

for some $k_{n}$ determined by the choice of normalization. A proof of this is provided for $N=2,3$ in Sections 4.1 and 4.2 respectively. We observe that $\Psi_{n} \sim P_{n+M-1}^{(\alpha, \beta)}(x)$, a result discussed further in Section 5.

A similar result holds if the boundary conditions are imposed only at $x=-1$; the roles of $\alpha$ and $\beta$ reverse leading to

$$
\Psi_{n}(x)=\left\{\begin{array}{l}
\sum_{i=1}^{M+n} c_{i}(n) P_{i-1}^{(\alpha, \beta+N)}(x), 1 \leq n \leq N-M, \\
\sum_{i=1}^{N+1} c_{i}(n) P_{M+n-i}^{(\alpha, \beta+N)}(x), n \geq N-M+1 .
\end{array}\right.
$$

A peculiar case arises in the determination of $c_{i}$, for $n \leq N-M$, when the boundary conditions do not supply enough constraints on the function. For instance, $\Psi_{1}(x)=c_{1}+c_{2} P_{1}^{(\alpha+N, \beta)}(x)$ is not determined (even up to a normalization) by the condition $\Psi_{n}^{\prime \prime}(1)=0$. Indeed, imposing this same condition on $\Psi_{2}(x)=c_{1}+c_{2} P_{1}^{(\alpha+N, \beta)}(x)+c_{3} P_{2}^{(\alpha+N, \beta)}(x)$ requires only that $c_{3}=0$, while orthogonality with respect to $\Psi_{1}$ still leaves three degrees of freedom among $\Psi_{1}$ and $\Psi_{2}$. While we could leave the undetermined $c_{i}$ arbitrary, we note that the space of linear combinations of these functions is also spanned by $\Psi_{1}(x)=1$, $\Psi_{2}(x)=P_{1}^{(\alpha, \beta)}(x)$, which are also orthogonal in the required manner of (8). It is therefore evident how one should proceed in the construction of an orthogonal basis set for such boundary conditions.

\subsection{Generalization}

By making use of the recurrence relation (67), (6) can be written in the symmetric form

$$
\Psi_{n}(x)=\sum_{i=1}^{2 N+1} c_{i} P_{M+n-i}^{(\alpha+N, \beta+N)}(x), \quad n \geq 2 N-M+1
$$

where we solve for the $2 N+1$ coefficients using the $M$ boundary conditions, one normalization condition, and $2 N-M$ orthogonality conditions. The first $2 N-M$ functions are "hard-wired" to be orthogonal and as before forced to satisfy the boundary conditions by using the standard Gram-Schmidt procedure. 
It is also apparent that the one-sided form of (9) with boundary conditions imposed only at $x=-1$ can be written in precisely the same way. It is therefore unsurprising (but not yet proven) that (10) is the most general representation required for any combination of $M$ boundary conditions of degree $N-1$ acting at either (or both) of the endpoints. In practice we find this to be the case.

\section{Integrals of Jacobi polynomials}

To discuss orthogonality of these generalized expansion sets we need integrals of various products of Jacobi polynomials of the form

$$
\int_{-1}^{1} P_{n}^{(\alpha+\mu, \beta+\nu)}(x) P_{m}^{(\alpha+\mu, \beta+\nu)}(x)(1+x)^{\beta}(1-x)^{\alpha} d x
$$

where $v=0$ in the one-sided case, and $v=\mu$ in the two-sided case. For the purposes of this paper, we need only consider positive integer values for $v$ and $\mu$, although we indicate the results that do hold for general (real) values. We begin by covering the one-sided case, whose associated integrals can be written in a simple closed form. We then turn to the two-sided case, where we have been unable to find a similarly simple expression, but results are found for specific cases.

Substituting

$$
P_{n}^{(\alpha, \beta)}(x)=\left(\begin{array}{c}
n+\alpha \\
\alpha
\end{array}\right){ }_{2} F_{1}\left(\begin{array}{c}
{[-n, 1+n+\alpha+\beta]} \\
{[\alpha+1]}
\end{array} ; \frac{1}{2}(1-x)\right)
$$

directly into (11), the integral immediately reduces to

$$
\begin{gathered}
2^{\alpha+\beta+1} \frac{\Gamma(\alpha+1) \Gamma(\beta+1)}{\Gamma(\alpha+\beta+2)}\left(\begin{array}{c}
m+\alpha+\mu \\
m
\end{array}\right)\left(\begin{array}{c}
n+\alpha+\mu \\
n
\end{array}\right) \\
\times \sum_{j=0}^{m} \frac{(-m)_{j}(\alpha+1)_{j}(1+m+\alpha+\beta+\mu+v)_{j}}{\Gamma(j+1)(\alpha+\beta+2)_{j}(\alpha+\mu+1)_{j}} \\
\quad \times{ }_{3} F_{2}\left(\begin{array}{c}
{[\alpha+j+1,-n, 1+n+\alpha+\beta+\mu+v]} \\
{[\alpha+\beta+j+2, \alpha+\mu+1]}
\end{array}\right) .
\end{gathered}
$$

In this case the Weber-Erdelyi transformation [17], gives

$$
\begin{aligned}
{ }_{3} F_{2}\left(\begin{array}{c}
{[\alpha+j+1,-n, 1+n+\alpha+\beta+\mu+v]} \\
{[\alpha+\beta+j+2, \alpha+\mu+1]}
\end{array} ;\right) \\
=\frac{\Gamma(j+\alpha+\beta+2) \Gamma(\mu+\alpha+1) \Gamma(\beta+1+n) \Gamma(-j+\mu+n)}{\Gamma(\mu+\alpha+1+n) \Gamma(j+\alpha+\beta+2+n) \Gamma(\beta+1) \Gamma(\mu-j)} \\
\quad \times{ }_{3} F_{2}\left(\begin{array}{c}
{[\alpha+j+1,-n, v]} \\
{[-\beta-v, 1+j-\mu-n]}
\end{array} ; 1\right) .
\end{aligned}
$$


For $v=0$, the hypergeometric function is unity. Collecting terms in $j$ in that case yields

$$
\begin{aligned}
& 2^{\alpha+\beta+1}\left(\begin{array}{c}
m+\alpha+\mu \\
m
\end{array}\right) \frac{(\mu)_{n}}{\Gamma(n+1)} \frac{\Gamma(\alpha+1) \Gamma(\beta+1+n)}{\Gamma(\alpha+\beta+n+2)} \\
& \quad \times{ }_{4} F_{3}\left(\begin{array}{c}
{[-m, 1-\mu, 1+\alpha, 1+m+\alpha+\beta+\mu]} \\
{[1+\mu+\alpha, \alpha+\beta+n+2,1-\mu-n]}
\end{array} ; 1\right), \quad m \leq n .
\end{aligned}
$$

Alternately, using Whipple's transformation [2] this can be rewritten as

$$
\begin{aligned}
& \frac{2^{\alpha+\beta+1} \Gamma(\alpha+1) \Gamma(n-m+\mu) \Gamma(2 \mu+\alpha+m) \Gamma(n+\beta+1)}{\Gamma(2 \mu+\alpha) \Gamma(\mu) \Gamma(n-m+1) \Gamma(m+1) \Gamma(n+\beta+2+\alpha)} \\
& \quad \times{ }_{4} F_{3}\left(\begin{array}{c}
{[-m, 1-\mu, n+\beta+1,-\mu+n-m+1]} \\
{[n-m+1,-m-2 \mu+1-\alpha, n+\beta+2+\alpha]}
\end{array} ; 1\right), \quad m \leq n .
\end{aligned}
$$

The symmetric identity follows as:

$$
\begin{aligned}
& \int_{-1}^{1} P_{n}^{(\alpha, \beta+\mu)} P_{m}^{(\alpha, \beta+\mu)}(1-x)^{\alpha}(1+x)^{\beta} d x \\
& =\frac{(-1)^{n-m} 2^{\alpha+\beta+1} \Gamma(\beta+1) \Gamma(n-m+\mu) \Gamma(2 \mu+\beta+m) \Gamma(n+\alpha+1)}{\Gamma(2 \mu+\beta) \Gamma(\mu) \Gamma(n-m+1) \Gamma(m+1) \Gamma(n+\beta+2+\alpha)} \\
& \quad \times{ }_{4} F_{3}\left(\begin{array}{c}
{[-m, 1-\mu, n+\alpha+1,-\mu+n-m+1]} \\
{[n-m+1,-m-2 \mu+1-\beta, n+\beta+2+\alpha]}
\end{array} ;\right)
\end{aligned}
$$

For applications here $\mu=M \in \mathbb{Z}_{>0}$ hence the ${ }_{4} F_{3}$ can be written as a finite sum over $k=1, \ldots M$ with $m$ and $n$ left as general arguments, although (14)-(16) all continue to hold for general $\mu$.

For the two-sided case, (13) cannot be evaluated in the same manner and we take a different tack, akin to the linearization of products of orthogonal polynomials (see e.g. [1]). By finding the coefficients $c_{m}$ in

$$
P_{n}^{(\alpha+\mu, \beta+\nu)}(x)=\sum_{m=0}^{n} c_{m} P_{m}^{(\alpha, \beta)}(x),
$$

direct substitution into (11) and invoking orthogonality of the standard Jacobi polynomials reduce the result to a single sum which, on assuming integer values for $\mu$ and $\nu$, turns out to be in a form that may be immediately evaluated with Gosper's algorithm [6].

Using the hypergeometric expansion (12) in

$$
\int_{-1}^{1} P_{n}^{(\alpha+\mu, \beta+v)}(x) P_{m}^{(\alpha, \beta)}(x)(1-x)^{\alpha}(1+x)^{\beta} \mathrm{d} x,
$$


produces a double sum over indices $j=0 \ldots m$ and $k=0 \ldots n$, with the integral over $x$ generating a factor

$$
\frac{2^{1+\alpha+\beta} \Gamma(\beta+1) \Gamma(j+k+\alpha+1)}{\Gamma(j+k+\alpha+\beta+2)} .
$$

The sum on $j$ then generates

$$
{ }_{3} F_{2}\left(\begin{array}{c}
{[-m, m+\alpha+\beta+1, k+\alpha+1]} \\
{[\alpha+1, k+\alpha+\beta+2]}
\end{array} ;\right) .
$$

We next use the Weber-Erdelyi transformation, reducing this balanced form to obtain

$$
\frac{\Gamma(k+\alpha+\beta+2) \Gamma(\alpha+1) \Gamma(k+1) \Gamma(-\beta)}{\Gamma(m+\alpha+1) \Gamma(m+k+\alpha+\beta+2) \Gamma(m+k-1) \Gamma(-m-\beta)}
$$

for $k \geq m$, and zero otherwise. Collecting all the factors for (18) we obtain

$$
\begin{aligned}
& \frac{2^{1+\alpha+\beta} \Gamma(1+m+\beta) \Gamma(n+\alpha+\mu+1) \Gamma(\alpha+1+m) \Gamma(m+n+v+\mu+1+\alpha+\beta)}{\Gamma(1+n+\alpha+\beta+\mu+v) \Gamma(1+m) \Gamma(m+\alpha+\mu+1) \Gamma(2 m+\alpha+\beta+2) \Gamma(n-m+1)} \\
& \quad \times{ }_{3} F_{2}\left(\begin{array}{c}
{[-n+m, m+\alpha+1, m+n+v+\mu+\alpha+\beta+1]} \\
{[2 m+\alpha+\beta+2, m+\alpha+\mu+1]}
\end{array}\right) .
\end{aligned}
$$

We apply the Weber-Erdelyi transformation once more to reexpress this as

$$
\begin{aligned}
& \frac{2^{1+\alpha+\beta} \Gamma(n-m+\mu) \Gamma(n+\beta+1) \Gamma(m+\alpha+1) \Gamma(m+n+v+\mu+1+\alpha+\beta)}{\Gamma(n+m+\alpha+\beta+2) \Gamma(\mu) \Gamma(n+\alpha+\beta+\mu+v+1) \Gamma(m+1) \Gamma(n-m+1)} \\
& \quad \times{ }_{3} F_{2}\left(\begin{array}{c}
{[v, m-n, m+\alpha+1]} \\
{[-n-\beta, m-n-\mu+1]}
\end{array} ; 1\right) .
\end{aligned}
$$

Taking account of the Jacobi normalization,

$$
\begin{aligned}
\int_{-1}^{1} & \left(P_{m}^{(\alpha, \beta)}(x)\right)^{2}(1-x)^{\alpha}(1+x)^{\beta} \mathrm{d} x \\
= & \frac{2^{1+\alpha+\beta} \Gamma(\alpha+1+m) \Gamma(1+m+\beta)}{(2 m+\alpha+\beta+1) \Gamma(1+m) \Gamma(m+\alpha+\beta+1)},
\end{aligned}
$$

it follows that the $c_{m}$ of (17) are

$$
\begin{aligned}
c_{m}= & \frac{\Gamma(n+\beta+1) \Gamma(n+m+\alpha+\beta+\mu+v+1) \Gamma(m+\alpha+\beta+1) \Gamma(n-m+\mu)}{\Gamma(m+\beta+1) \Gamma(n+\alpha+\beta+\mu+v+1) \Gamma(n+m+\alpha+\beta+2) \Gamma(n-m+1) \Gamma(\mu)} \\
& \times(2 m+\alpha+\beta+1){ }_{3} F_{2}\left(\begin{array}{c}
{[v, m-n, m+\alpha+1]} \\
{[-n-\beta, m-n-\mu+1]}
\end{array} ; 1\right) .
\end{aligned}
$$


The key to progress here is that once we restrict $(\mu, \nu)=(M, N) \in \mathbb{Z}_{>0}^{2}$, the ${ }_{3} F_{2}$ can be summed in closed form by Gosper's algorithm [6], producing a polynomial form in $m$ multiplied by a quotient composed of gamma functions.

Returning to the evaluation of (11) with $(\mu, v)=(M, N)$, we see that on substituting (17), the resulting sum terminates at the lesser of $n$ and $m$ and in the following we use the convention that the indices are ordered with $m \leq n$. (This is the reason that, although the integral itself is symmetric under interchange of the indices, the formulas that follow are not.)

It is therefore possible to find a closed form expression for (11) for general $(\alpha, \beta)$ and any given pair $(\mu, \nu)=(M, N)$ but, guided by present need, we focus on the symmetric case of $\beta=\alpha$ and $N=M$. Then

$$
\begin{aligned}
c_{m}= & 4^{N-1}\left(1+(-1)^{m+n}\right)(2 m+2 \alpha+1) \frac{\Gamma((n+m+1) / 2+\alpha+N)}{\Gamma((n+m+3) / 2+\alpha)} \\
& \times \frac{\Gamma((n-m) / 2+N) \Gamma(m+2 \alpha+1) \Gamma(n+\alpha+N+1)}{\Gamma((n-m) / 2+1) \Gamma(m+\alpha+1) \Gamma(n+2 \alpha+2 N+1) \Gamma(N)} .
\end{aligned}
$$

(The expression for the one-sided $c_{m}$ with $\mu=N, v=0$, is trivial from the previous expression as the ${ }_{3} F_{2}$ simply drops out.) Note that, for any positive integers $j$ and $k$,

$$
\int_{-1}^{1} P_{2 j+1}^{(\alpha+N, \alpha+N)}(x) P_{2 k}^{(\alpha+N, \alpha+N)}(x)\left(1-x^{2}\right)^{\alpha} d x=0,
$$

as follows from noting the parity of the polynomials [16, p. 59]. It is therefore to be understood the formulas that follow hold for $\bmod (n+m, 2)=0$, otherwise the integral is zero.

For $N=M=2$ this prescription yields for (11)

$$
\begin{aligned}
& \frac{2^{2 \alpha+2} \Gamma(m+\alpha+3) \Gamma(n+\alpha+3)}{(\alpha+1)_{3} \Gamma(m+1) \Gamma(n+2 \alpha+5)}\left[(\alpha+3) n^{2}-(\alpha+1) m^{2}\right. \\
& \quad-(\alpha+1)(2 \alpha+5) m+(\alpha+3)(2 \alpha+5) n+2(\alpha+3)(2 \alpha+3)],
\end{aligned}
$$

(where we use the Pochhammer notation $(a)_{n}=a(a+1) \ldots(a+n-1)$ ) and for $N=M=3$,

$$
\begin{aligned}
& \frac{2^{2 \alpha+1}}{(\alpha+1)} \Gamma(m+\alpha+4) \Gamma(n+\alpha+4) \\
& \times {\left[n^{4}(\alpha+5)(\alpha+4)-2 n^{2} m^{2}(\alpha+5)(\alpha+1)+m^{4}(\alpha+2)(\alpha+1)\right.} \\
&+2 n^{3}(\alpha+5)(\alpha+4)(2 \alpha+7)-2\left(n^{2} m+n m^{2}\right)(\alpha+5)(\alpha+1)(2 \alpha+7) \\
&+2 m^{3}(\alpha+2)(\alpha+1)(2 \alpha+7)+n^{2}(\alpha+5)(\alpha+4)\left(4 \alpha^{2}+40 \alpha+71\right) \\
&-2 n m(\alpha+5)(\alpha+1)(2 \alpha+7)^{2}+m^{2}(\alpha+1)\left(4 \alpha^{3}+24 \alpha^{2}+19 \alpha-38\right) \\
&+2 n(\alpha+5)(\alpha+4)(2 \alpha+7)(6 \alpha+11)-2 m(\alpha+1)(2 \alpha+7) \\
&\left.\times\left(6 \alpha^{2}+43 \alpha+68\right)+8(\alpha+5)(\alpha+4)(2 \alpha+3)(2 \alpha+5)\right] .
\end{aligned}
$$


Finally for $N=M=4$ the result for general $\alpha$ is too involved to warrant reproducing here. Instead we provide only the reduced forms for the common cases of $\alpha=-1 / 2,0,1 / 2$, namely

$$
\begin{aligned}
\frac{16}{135135} & \frac{\Gamma(m+9 / 2) \Gamma(n+9 / 2)}{\Gamma(n+8) \Gamma(m+1)} \\
\times[ & 429 n^{6}-143 m^{2} n^{4}+39 m^{4} n^{2}-5 m^{6}+10296 n^{5}-1144 n^{4} m \\
- & 2288 n^{3} m^{2}+624 n^{2} m^{3}+312 n m^{4}-120 m^{5}+99528 n^{4}-18304 n^{3} m \\
- & 11440 n^{2} m^{2}+4992 n m^{3}-152 m^{4}+494208 n^{3}-111488 n^{2} m \\
- & 18304 n m^{2}+10368 m^{3}+1324752 n^{2}-306176 n m+10096 m^{2} \\
+ & 1812096 n-332928 m+988416] \\
\frac{1}{1260} & \frac{(m+1)_{4}}{(n+5)_{4}} \\
\times & {\left[35 n^{6}-21 n^{4} m^{2}+7 n^{2} m^{4}-m^{6}+945 n^{5}-189 n^{4} m-378 n^{3} m^{2}\right.} \\
& +126 n^{2} m^{3}+63 n m^{4}-27 m^{5}+10325 n^{4}-3402 n^{3} m-2002 n^{2} m^{2} \\
& +1134 n m^{3}-71 m^{4}+58275 n^{3}-23121 n^{2} m-2709 n m^{2}+2367 m^{3} \\
& \left.+178640 n^{2}-70308 n m+4752 m^{2}+280980 n-82620 m+176400\right](24)
\end{aligned}
$$

$$
\frac{64}{405405} \frac{\Gamma(m+11 / 2) \Gamma(n+11 / 2)}{\Gamma(n+10) \Gamma(m+1)}
$$$$
\times\left[143 n^{6}-117 n^{4} m^{2}+45 n^{2} m^{4}-7 m^{6}+4290 n^{5}\right.
$$$$
-1170 n^{4} m-2340 n^{3} m^{2}+900 n^{2} m^{3}+450 n m^{4}-210 m^{5}+52052 n^{4}
$$$$
-23400 n^{3} m-12960 n^{2} m^{2}+9000 n m^{3}-820 m^{4}+326040 n^{3}
$$$$
-174600 n^{2} m-12600 n m^{2}+18600 m^{3}+1107392 n^{2}-576000 n m
$$$$
\left.+56384 m^{2}+1921920 n-716160 m+1317888\right]
$$

respectively.

Gosper's algorithm is quite general and therein lies its power. But for the same reason, our results for the two-sided case above stand in sharp contrast to the terse general result in (14) and it is not reasonable to expect a comparable economy could emerge from our approach thus far. Nonetheless the twosided answer for (11) must reflect all the recurrence properties of the Jacobi 
polynomials and this implies constraints on form. In Section 4.3 we point out salient properties of the matrix whose entries are defined by (11) with $(\mu, v)=(M, N)$ that clarify the algebraic structure of the integral, which leads us to a suitable generalization of (14).

A final comment: So far we have deemed it expedient (mainly for typographical purposes) to leave the $c_{i}$ defined only up to a multiplicative constant, equivalent to leaving the resulting basis set unnormalized. However, the natural normalization for the basis set is

$$
\int_{-1}^{1}\left[\Psi_{n}(x)\right]^{2}(1-x)^{\alpha}(1+x)^{\beta} \mathrm{d} x=1,
$$

which is easily enough implemented, although the resulting expressions for general $n$ are extremely lengthy and are hence omitted throughout.

\section{Auto-orthogonality}

We are now in a position to prove the validity of the construction given in Section 2. Our approach is to look within the space of auto-orthogonal polynomials and show that there are sufficient degrees of freedom in order that we can satisfy the required boundary conditions. In the following two sections, we prove the two simplest cases of one-sided boundary conditions of first and second order. Lastly we indicate how a general proof for arbitrary boundary conditions for both the one-sided and two sided constructions might proceed.

\subsection{One-sided first order boundary conditions}

Let us begin by taking the simplest possible (nontrivial) case, that of a onesided construction with first order boundary conditions, corresponding to $N=2$. We will prove here that the construction of Section 2.1, involving just three Jacobi polynomials, suffices to generate an auto-orthogonal set. Without loss of generality, we may assume that we have only a single boundary condition $(M=1)$, since $M=2$ amounts to the simple mapping $n \rightarrow n+1$, $m \rightarrow m+1$ in the following analysis. Defining the vector $\mathbf{c}(n)=\left[c_{1}(n)\right.$, $\left.c_{2}(n), c_{3}(n)\right]^{T}$, showing the existence of an auto-orthogonal set amounts to finding the solutions of

$$
\mathbf{c}^{T}(m) \mathbf{A}_{3}^{(2,0)} \mathbf{c}(n)=0,
$$

where $\mathbf{A}_{3}^{(2,0)}$ is a matrix (whose superscripts indicate $(\mu, v)$ from Section 3) with entries $a_{i, j}$ defined by

$$
a_{i, j}=\int_{-1}^{1} P_{n+1-i}^{(\alpha+2, \beta)}(x) P_{m+1-j}^{(\alpha+2, \beta)}(x)(1-x)^{\alpha}(1+x)^{\beta} \mathrm{d} x \quad i, j=1,2,3 .
$$


Given the form of the integral derived from (14), the matrix $\mathbf{A}_{3}^{(2,0)}$ can be written as the sum of two outer products

$$
\begin{aligned}
\mathbf{A}_{3}^{(2,0)}= & -\frac{2^{\alpha+\beta+1}}{(\alpha+1)(\alpha+2)(\alpha+3)} \frac{\Gamma(m+1+\alpha) \Gamma(n-1+\beta)}{\Gamma(m+1) \Gamma(n+3+\alpha+\beta)} \\
& \times\left[\left(\mathbf{u}_{0} * \mathbf{u}_{1}\right) \mathbf{v}_{0}^{T}+\mathbf{u}_{0}\left(\mathbf{v}_{0} * \mathbf{v}_{1}\right)^{T}\right]
\end{aligned}
$$

where

$$
\begin{aligned}
\mathbf{u}_{0}= & {[(2+\alpha+m)(1+\alpha+m), m(1+\alpha+m), m(m-1)]^{T}, } \\
\mathbf{v}_{0}= & {[(n+\beta)(n-1+\beta),(n-1+\beta)(n+\beta+2+\alpha),} \\
& (n+\beta+2+\alpha)(n+\beta+1+\alpha)]^{T}, \\
\mathbf{u}_{1}= & (\alpha+1)[m(\alpha+\beta+m+3),(m-1)(\alpha+\beta+m+2), \\
& (m-2)(\alpha+\beta+m+1)]^{T}-(\alpha+3)[1,1,1]^{T}, \\
\mathbf{v}_{1}= & (\alpha+3)[(-n)(n+3+\alpha+\beta),(1-n)(n+2+\alpha+\beta), \\
& (2-n)(n+1+\alpha+\beta)]^{T} .
\end{aligned}
$$

and we use $*$ to denote the element-by-element product of vectors. Since $\mathbf{A}_{3}^{(2,0)}$ is rank deficient by one, it has determinant zero.

Although Eq. 27 is quadratic, because of the separation in $n$ and $m$ dependence, solutions lie in one of three classes of linear equations. These take the form of: left singular vectors, $\mathbf{c}=\mathbf{l}$, right singular vectors, $\mathbf{c}=\mathbf{r}$, and "general" mixed solutions.

The simplest solution is the right singular vector, which is a function of $n$ only, and effects the cancellation needed in (27) purely from the right. It corresponds to the homogeneous system

$$
\mathbf{v}_{0}^{T}(n) \mathbf{c}(n)=0, \quad\left(\mathbf{v}_{0} * \mathbf{v}_{1}\right)^{T} \mathbf{c}(n)=0
$$

which has unnormalized solution $\mathbf{r}$ with components given by

$$
\begin{aligned}
& r_{1}=(2 n+\alpha+\beta)(n+\beta+2+\alpha)(n+\beta+1+\alpha), \\
& r_{2}=-2(n+\beta)(n+\beta+1+\alpha)(2 n+1+\alpha+\beta), \\
& r_{3}=(n+\beta)(n-1+\beta)(2 n+\alpha+2+\beta) .
\end{aligned}
$$

In fact, by inspecting (66), this is simply $P_{n}^{(\alpha, \beta)}(x)$. It follows that no particular boundary condition is satisfied at $x=1$, a property that may be verified by evaluating $f(1)$ and $f^{\prime}(1)$ and noting that they are incommensurate functions of $n$ so that no linear combination, independent of $n$, can be made to vanish. 
Defining $\Psi_{1}=1, \Psi_{2}=P_{1}^{(\alpha, \beta)}(x)$, we complete the orthogonal family, which is simply the standard Jacobi polynomials themselves.

The left singular vector, being a function of $m$ only and effecting the required cancellation from the left, satisfies

$$
\mathbf{c}(m)^{T} \mathbf{u}_{0}(m)=0, \quad \mathbf{c}(m)^{T}\left(\mathbf{u}_{0} * \mathbf{u}_{1}\right)=0,
$$

leading to the unnormalized solution I where

$$
\begin{aligned}
& l_{1}=m(-1+m)(2 m+\alpha+\beta), \\
& l_{2}=-2(-1+m)(m+2+\alpha)(2 m+\alpha+1+\beta), \\
& l_{3}=(m+2+\alpha)(m+1+\alpha)(2 m+\alpha+2+\beta) .
\end{aligned}
$$

In contrast to the right singular vector that satisfies no boundary conditions, the left singular vector satisfies two: $f(1)=f^{\prime}(1)=0$. This family of orthogonal solutions can alternatively be written as $\Psi_{n}=(1-x)^{2} P_{n-2}^{(\alpha+4, \beta)}(x)$.

In addition to these rather special right and left solutions, (28) also admits solutions with one degree of freedom:

$$
\begin{gathered}
\mathbf{v}_{0}^{T}(n) \mathbf{c}(n)=\mu\left(\mathbf{v}_{0}(n) * \mathbf{v}_{1}(n)\right)^{T} \mathbf{c}(n), \\
\mathbf{c}(m)^{T} \mathbf{u}_{0}(m)=-\mu \mathbf{c}(m)^{T}\left(\mathbf{u}_{0}(m) * \mathbf{u}_{1}(m)\right) .
\end{gathered}
$$

The lowest order polynomial that allows for arbitrary $\mu$ is degree seven, with the corresponding components of $\mathbf{c}$ given by

$$
\begin{aligned}
& c_{1}=n(n+2+\alpha+\beta)(2 n+\alpha+\beta) p_{4}^{(1)}(n ; \alpha, \beta, \mu), \\
& c_{2}=-(2 n+1+\alpha+\beta) p_{6}(n ; \alpha, \beta, \mu), \\
& c_{3}=(n-1+\beta)(n+1+\alpha)(2 n+2+\alpha+\beta) p_{4}^{(2)}(n ; \alpha, \beta, \mu),
\end{aligned}
$$

where we use $p_{k}(n ; \alpha, \beta, \mu)$ to denote an irreducible polynomial in $n$ of degree $k .^{3}$

The expansion in (34) satisfies

$$
2(\alpha+1)(\alpha+3) \mu f^{\prime}(1)+(\mu(\alpha+2+\beta)(\alpha+3)-1) f(1)=0,
$$

and hence allows for a boundary condition with an arbitrary linear combination of the function and its first derivative for any $\alpha, \beta>-1$. The first element in the set is

$$
1+\frac{1-\mu(\alpha+3)(\alpha+2+\beta)}{\mu(\alpha+3)(3 \alpha+4+\beta)-1} x .
$$

\footnotetext{
${ }^{3}$ Note the identical form to that derived in Appendix B.
} 
Thus the general solution can deal with any single boundary condition involving the function and its first derivative at $x=1$. If instead two nondegenerate conditions are specified, these must reduce to $f(1)=f^{\prime}(1)=0$ that is associated with the left singular vector. It is not possible to specify more than two non-degenerate such boundary conditions. It is of interest to see whether the general solution allows for boundary conditions of higher order. Using (69), one finds that there is no simple relation of the second derivative of the general solution to either the function or its first derivative, so boundary conditions for an expansion of this order may not include $f^{\prime \prime}$. Moreover the result for $f^{\prime \prime}$ cannot be made to vanish for any value of $\mu$ independent of $n$, and hence even $f^{\prime \prime}(1)=0$ is disallowed. This is consistent with the construction given in Section 2.1 that a second-order boundary conditions requires $N=3$.

The careful reader may have observed that there are, ostensibly, more than three solutions of (27). For instance, one possibility is a right singular vector for the first outer product and left for the second,

$$
\mathbf{v}_{0}^{T}(n) \mathbf{c}(n)=0, \quad \mathbf{c}(m)^{T} \mathbf{u}_{0}(m)=0 .
$$

However, this corresponds to $\mu=0$ (i.e., $f(1)=0$ ), while the reverse relation

$$
\left(\mathbf{v}_{0} * \mathbf{v}_{1}\right)^{T}(n) \mathbf{c}(n)=0, \quad \mathbf{c}(m)^{T}\left(\mathbf{u}_{0} * \mathbf{u}_{1}\right)(m)=0,
$$

satisfies $(\alpha+\beta+2) f=2(\alpha+1) f^{\prime}=0$ and corresponds to $\mu \rightarrow \pm \infty$.

We lastly comment on the asymptotic behavior of these three distinct types of solution. As $n \rightarrow \infty$, the ratio of the coefficients in both (31) and (30) is $[1,-2,1]$. Additionally, it transpires that the same ratio applies to (34). Thus in each case, $\Psi_{n} \sim P_{n}^{(\alpha, \beta)}(x)$, as indicated in the introduction. Higher order corrections, which bear on the distinction between uniform convergence of this limit in the interior and the nonuniform limit as $x \rightarrow 1$, are discussed in Section 5 .

\subsection{One-sided second-order boundary conditions}

Paralleling the development above rapidly becomes algebraically involved but wholly unexceptional insofar as the dependence on general $(\alpha, \beta)$ is concerned. Consequently we restrict attention in this section to the Chebyshev case, $\alpha=\beta=-1 / 2$, since the main point here is the extension of general solutions to higher order outer products and their relation to boundary conditions.

Second order boundary conditions correspond to $N=3$, and the outer product for the one-sided problem (boundary conditions only at $x=1$ ) can be written as:

$$
\begin{aligned}
\mathbf{A}_{4}^{(3,0)}= & 2 \frac{\Gamma(n-5 / 2) \Gamma(m+1 / 2)}{\Gamma(n+3) \Gamma(m+1)} \\
& \times\left[\left(\mathbf{u}_{0} * \mathbf{u}_{1}\right) \mathbf{v}_{0}^{T}+\mathbf{u}_{0}\left(\mathbf{v}_{0} * \mathbf{v}_{1}\right)^{T}+\left(\mathbf{u}_{0} * \mathbf{u}_{2}\right)\left(\mathbf{v}_{0} * \mathbf{v}_{2}\right)^{T}\right]
\end{aligned}
$$


where

$$
a_{i, j}=\int_{-1}^{1} P_{n+1-i}^{(5 / 2,-1 / 2)}(x) P_{m+1-j}^{(5 / 2,-1 / 2)}(x)\left(1-x^{2}\right)^{-1 / 2} \mathrm{~d} x, \quad i, j=1,2,3,4
$$

and, as before, we have assumed that there is only one boundary condition $(M=1)$. The components are given by

$$
\begin{aligned}
\mathbf{u}_{0}= & \frac{1}{8}[(2 m+5)(2 m+3)(2 m+1), 2 m(2 m+3)(2 m+1), \\
& 4 m(2 m+1)(m-1), 8(m-2)(m-1) m]^{T}, \\
\mathbf{v}_{0}= & \frac{1}{1260}[(2 n-5)(2 n-3)(2 n-1), 2(2 n-5)(n+2)(2 n-3), \\
& 4(2 n-5)(n+2)(n+1), 8 n(n+1)(n+2)]^{T}, \\
\mathbf{u}_{1}= & {[m(m-1)(m+4)(m+3),(m-1)(m-2)(m+3)(m+2),} \\
& \left.u_{1}^{2}(-n), u_{1}^{1}(-n)\right]^{T}, \\
\mathbf{v}_{1}= & 21 n\left[(n+3)(n+2)(n+1),(n-1)(n+2)(n+1), v_{1}^{2}(-n), v_{1}^{1}(-n)\right]^{T}, \\
\mathbf{u}_{2}= & {\left[42-18 m-6 m^{2}, 54-6 m-6 m^{2}, u_{2}^{2}(-n), u_{2}^{1}(-n)\right]^{T}, } \\
\mathbf{v}_{2}= & {\left[(n+2)(n+1), n(n+1), v_{2}^{2}(-n), v_{2}^{1}(-n)\right]^{T}, }
\end{aligned}
$$

where $\mathbf{u}_{1}, \mathbf{u}_{2}, \mathbf{v}_{1}$ and $\mathbf{v}_{2}$ have the symmetry property that their individual components satisfy $w_{k}(n)=w_{5-k}(-n)$ for $k=1,2,3,4$. We use this for concision in the expressions above. As in the previous case, there are right and left singular vectors that correspond to, respectively, the Jacobi polynomials $P_{n}^{(-1 / 2,-1 / 2)}(x)$ (satisfying no boundary condition) and $(1-x)^{3} P_{n-3}^{(11 / 2,-1 / 2)}(x)$ (satisfying $\left.f(1)=f^{\prime}(1)=f^{\prime \prime}(1)=0\right)$.

The general solutions admit two free parameters and fall into two categories that we term "full" and "partial". The former solutions are those satisfying

$$
\begin{array}{r}
\left(\mathbf{u}_{0} * \mathbf{u}_{1}\right)^{T} \mathbf{c}_{1}(m)=\mu_{1} \mathbf{u}_{0}^{T} \mathbf{c}_{1}(m), \\
\left(\mathbf{u}_{0} * \mathbf{u}_{2}\right)^{T} \mathbf{c}_{1}(m)=\mu_{2} \mathbf{u}_{0}^{T} \mathbf{c}_{1}(m), \\
\left(\mathbf{v}_{0} *\left(\mathbf{v}_{1}+\mu_{2} \mathbf{v}_{2}\right)\right)^{T} \mathbf{c}_{1}(n)=-\mu_{1} \mathbf{v}_{0}^{T} \mathbf{c}_{1}(n),
\end{array}
$$

while the partial solutions satisfy

$$
\begin{aligned}
\left(\mathbf{v}_{0} * \mathbf{v}_{1}\right)^{T} \mathbf{c}_{2}(n) & =\mu_{1} \mathbf{v}_{0}^{T} \mathbf{c}_{2}(n), \\
\left(\mathbf{v}_{0} * \mathbf{v}_{2}\right)^{T} \mathbf{c}_{2}(n) & =\mu_{2} \mathbf{v}_{0}^{T} \mathbf{c}_{2}(n), \\
\left(\mathbf{u}_{0} *\left(\mathbf{u}_{1}+\mu_{2} \mathbf{u}_{2}\right)\right)^{T} \mathbf{c}_{2}(m) & =-\mu_{1} \mathbf{u}_{0}^{T} \mathbf{c}_{2}(m) .
\end{aligned}
$$


The distinction between these sets will shortly become apparent. They have auto-orthogonal solutions

$$
\begin{array}{r}
\mathbf{c}_{1}=\left(\begin{array}{c}
(2 n-5)(2 n+1)^{2}(2 n+3)(n+1) p_{8}(n) \\
-2(2 n-1)(2 n+3)(n+1) p_{10}(n) \\
4(2 n+1)(n-1)^{2} p_{10}(-n) \\
-8 n(2 n-1)(n+2)(n-1)^{2} p_{8}(-n)
\end{array}\right) \\
\mathbf{c}_{2}=\left(\begin{array}{c}
(2 n-5)(2 n+1)^{2}(2 n-3) \tilde{p}_{8}(n) \\
-2(2 n-1)(2 n-3) \tilde{p}_{10}(n) \\
4(2 n+1)(n-1) \tilde{p}_{10}(-n) \\
-8 n(2 n-1)(n+2)(n-1) \tilde{p}_{8}(-n)
\end{array}\right)
\end{array}
$$

where the full solutions are listed on the left and the partial solutions on the right. Note the antisymmetric pairing of the irreducible factors of degree $k$ denoted $p_{k}$ or $\tilde{p}_{k}$, which depend on the two unknown parameters $\mu_{1}, \mu_{2}$. Additionally, both $\mathbf{c}_{1}$ and $\mathbf{c}_{2}$ have coefficient ratios given by $[1,-3,3,-1]$ as $n \rightarrow \infty$. For the full solutions, we impose a second-order boundary condition of the form

$$
f(1)+\lambda_{1} f^{\prime}(1)+\lambda_{2} f^{\prime \prime}(1)=0,
$$

which results in the condition

$$
2 \lambda_{2} \mu_{1}-3 \lambda_{1} \mu_{2}+126\left(\lambda_{1}+1\right)=0 .
$$

We can impose a second boundary condition of the same form, with constants $\lambda_{3}, \lambda_{4}$, and are then led to

$$
\mu_{1}=\frac{63\left(\lambda_{1}-\lambda_{3}\right)}{\lambda_{2} \lambda_{3}-\lambda_{4} \lambda_{1}}, \quad \mu_{2}=42\left[1+\frac{\lambda_{2}-\lambda_{4}}{\lambda_{2} \lambda_{3}-\lambda_{4} \lambda_{1}}\right] .
$$

We write the first basis vector as $1+c_{1}(1-x)+c_{2}(1-x)^{2}$ and enforce the same boundary conditions hence

$$
c_{1}=\frac{\lambda_{2}-\lambda_{4}}{\lambda_{2} \lambda_{3}-\lambda_{4} \lambda_{1}}, \quad c_{2}=\frac{\lambda_{1}-\lambda_{3}}{2\left(\lambda_{2} \lambda_{3}-\lambda_{4} \lambda_{1}\right)} .
$$

Lastly, we confirm that the general element is orthogonal to this, for which we can use the simple result that

$$
\int_{-1}^{1}(1-x)^{k} P_{n}^{(5 / 2,-1 / 2)}(x) \frac{\mathrm{d} x}{\sqrt{1-x^{2}}}=2^{k} \frac{\Gamma(k+1 / 2) \Gamma(n+1 / 2) \Gamma(n-k+3)}{\Gamma(3-k) \Gamma(n+1) \Gamma(n+1+k)},
$$

and indeed the orthogonality holds. It is notable that the four degrees of freedom in the boundary conditions given by $\lambda_{1,2,3,4}$ collapse to just two parameters $\mu_{1}$ and $\mu_{2}$ in (38), and means that any given basis function satisfies an infinite number of conditions. However, this degeneracy only reflects that an arbitrary linear combination of the given homogeneous boundary conditions must also be satisfied and does not impose any additional constraints on the basis set. 
Turning now to the second class of solutions, those that we have termed partial, in contrast with the full solutions, only one second order boundary condition is satisfied, namely

$$
f(1)-\frac{42 \mu_{2}}{\mu_{1}+42 \mu_{2}} f^{\prime}(1)+\frac{63}{\mu_{1}+42 \mu_{2}} f^{\prime \prime}(1)=0 .
$$

Note here that only the trilinear combination vanishes in contrast with the full solution where, because two linearly independent second order boundary conditions are satisfied, we have the two independent relations $f(1)=$ $42 f^{\prime}(1) /\left(42-\mu_{2}\right)=63 f^{\prime \prime}(1) / \mu_{1}$.

The full and partial solutions augmented by the left singular vector span every possible choice of boundary conditions at $x=1$ up to and including second order; additionally an expansion in any of these solutions converges exponentially, as we discuss in Section 5.2.

\subsection{The general case}

We now remark on some general properties of the outer product representation for the one-sided case, and indicate how the proof of auto-orthogonality for the cases $N=2,3$ might be extended to arbitrary $N$. We determine a structure for the associated outer product representation and argue that an understanding of how the outer product is composed is intimately linked to the algebraic form of the one- and two-sided integrals.

\subsubsection{The one-sided outer product}

We begin by pointing out that, in the one-sided case, the right and left singular vectors generalize in an obvious manner:

$$
P_{n}^{(\alpha, \beta)}(x), \quad(1-x)^{N} P_{n-N}^{(\alpha+2 N, \beta)}(x) .
$$

We show this in two stages: by demonstrating that such polynomials are particular auto-orthogonal cases of (6), and then showing that each is, in the relevant sense, a singular vector. The first property follows because both define functions that are orthogonal with respect to the weight function $(1-x)^{\alpha}(1+x)^{\beta}$, and additionally may be expanded in terms of $N+1$ coefficients of Jacobi polynomials $P_{i}^{(\alpha+N, \beta)}(x)$ of neighboring degree by using (64) and (66). Additionally, they satisfy two additional identities:

$$
\begin{gathered}
\int_{-1}^{1} P_{m}^{(\alpha+N, \beta)}(x) P_{n}^{(\alpha, \beta)}(x)(1-x)^{\alpha}(1+x)^{\beta} d x=0, \quad m<n, \\
\int_{-1}^{1}(1-x)^{N} P_{m-N}^{(\alpha+2 N, \beta)}(x) P_{n}^{(N+\alpha, \beta)}(x)(1-x)^{\alpha}(1+x)^{\beta} d x=0, \\
m-N<n .
\end{gathered}
$$


To prove these, we note that in $(40), P_{m}^{(N+\alpha, \beta)}(x)$ is a polynomial of degree $m$, which can be expanded using (17) and the result follows by standard orthogonality. In (41), we similarly expand $P_{m-N}^{(\alpha+2 N, \beta)}(x)$ as a sum over $P_{i}^{(\alpha+N, \beta)}(x)$. An immediate consequence is that, with $m<n$ :

$$
\begin{aligned}
& \int_{-1}^{1}\left(\sum_{j=1}^{N+1} c_{j} P_{m+1-j}^{(\alpha+N, \beta)}(x)\right) P_{n}^{(\alpha, \beta)}(x)(1-x)^{\alpha}(1+x)^{\beta} d x=0, \\
& \int_{-1}^{1}(1-x)^{N} P_{m-N}^{(\alpha+2 N, \beta)}(x)\left(\sum_{i=1}^{N+1} c_{i} P_{n+1-i}^{(\alpha+N, \beta)}(x)\right)(1-x)^{\alpha}(1+x)^{\beta} d x=0,
\end{aligned}
$$

so that the two sets of functions, represented in coefficient space as $\mathbf{c}=\mathbf{r}$ or $\mathbf{c}=\mathbf{l}$, do indeed effect the cancellation required in

$$
\mathbf{c}^{T}(m) \mathbf{A}_{N+1}^{(N, 0)} \mathbf{c}(n)=0,
$$

and so define right and left singular vectors respectively. It follows that $\mathbf{A}_{N+1}^{(N, 0)}$ must always be rank defective by one. Note that the asymmetric relation $m<n$ is consistent with that required in (14).

It is helpful at this point to slightly alter the notation of the preceding sections in suggesting that the outer product expansion for the one-sided case can be put in the general form

$$
\mathbf{A}_{N+1}^{(N, 0)}=\Phi(m, n) \sum_{k=0}^{N-1} \mathbf{u}^{(k)}(m) \mathbf{v}^{(N-1-k) T}(n)
$$

where the vectors $\mathbf{u}$ and $\mathbf{v}$ must be representable in terms of "annihilators" of $\mathbf{I}$ and $\mathbf{r}$ respectively, defined by

$$
\tilde{\mathbf{u}}^{(k) T} \mathbf{l}=0 \quad \text { and } \quad \tilde{\mathbf{v}}^{(k) T} \mathbf{r}=0, \quad k=0 \ldots(N-1),
$$

respectively. We have already seen this in the preceding sections where (in the former notation) both $\mathbf{u}^{(0) T}$ and $\left(\mathbf{u}^{0} * \mathbf{u}^{k}\right)^{T}$ annihilate $\mathbf{l}$ and both $\mathbf{v}^{(0) T}$ and $\left(\mathbf{v}^{0} * \mathbf{v}^{k}\right)^{T}$ annihilate $\mathbf{r}$, for $k=1,2$. With regard to notation, $\mathbf{u}$ and $\mathbf{v}$ are not to be confused with the standard singular value decomposition. In particular, the matrices are not unitary; it is readily confirmed from the forms in the previous two sections that the vectors are not orthogonal.

For general $N$, we find a natural form for components of the first set of annihilators with $\alpha=\beta=-1 / 2$ to be:

$$
\begin{aligned}
\tilde{u}_{N+1-j}^{(k)}= & \frac{\Gamma(m+j+1 / 2) \Gamma(m+j+\sigma) \Gamma(m+j+k-\sigma-N+1)}{\Gamma(m-N+j+1) \Gamma(m+j+\sigma-k) \Gamma(m+j-\sigma-N+1)} \\
& \left\{\begin{array}{l}
j=0 \ldots N, \\
k=0 \ldots(N-1),
\end{array}\right.
\end{aligned}
$$


for arbitrary $\sigma$. We argue that, up to an overall scale factor, each vector $\mathbf{u}^{(k)}$ in the outer product decomposition is composed of a particular linear combination of these $\tilde{\mathbf{u}}^{(k)}$. Though it is easy enough to recover the particular results of the two preceding sections, this prescription must ultimately derive from the properties of (14) but we have been unable to establish such a general relation (except for $\mathbf{u}^{(0)}$, which is found by setting $k=\sigma=0$ ). Nonetheless, this line of development does suggest the intimate relation between the index recurrence relation (66) and the consequent form that the integral of the product of the corresponding Jacobi polynomials must take, as we examine next.

\subsubsection{The two-sided outer product}

In the two-sided case, the right and left singular vectors are the requisite representations of

$$
P_{n}^{(\alpha, \beta)}(x), \quad(1-x)^{N}(1+x)^{N} P_{n-2 N}^{(\alpha+2 N, \beta+2 N)}(x)
$$

a fact that is easily proven by applying similar arguments as those for the onesided case above. As before, it follows that the matrix $\mathbf{A}_{2 N+1}^{(N, N)}$ is rank defective by one.

Similarly, (44) generalizes to

$$
\begin{aligned}
& \tilde{u}_{2(N-j)+1}^{(k)} \\
& =\frac{\Gamma(m+2 j+\sigma) \Gamma(m+2 j+2 N+k+1-\sigma) \Gamma(m+2 j+N+1 / 2)}{\Gamma(m+2 j+\sigma-k) \Gamma(m+2 j+2 N+1-\sigma) \Gamma(m+2 j+1)}, \\
& \tilde{v}_{2(N-j)+1}^{(k)} \\
& =\frac{\Gamma(n+2 j+2 N+\tau) \Gamma(n+2 j+1-\tau+k) \Gamma(n+2 j+N+1 / 2)}{\Gamma(n+2 j+2 N+\tau-k) \Gamma(n+2 j+1-\tau) \Gamma(n+2 j+2 N)}, \\
& \tilde{u}_{2 j}^{(k)}=\tilde{v}_{2 j}^{(k)}=0, \quad\left\{\begin{array}{l}
j=0 \ldots N, \\
k=0 \ldots(N-1),
\end{array}\right.
\end{aligned}
$$

again for arbitrary $\sigma$ and $\tau$. Note that the case of $\alpha=\beta$ is special in that each of the left and right singular vectors has a definite symmetry and so in addition to the above there is a complementary set of $N$ vectors, for which the elements with subscript $2 j$ are nonzero and those with $2 j+1$ vanish. Whichever set is adopted, the matrix $\mathbf{A}_{2 N+1}^{(N, N)}$ has a checkerboard pattern: alternate entries are zero based on symmetry properties of the integral noted previously in Section 3.

If $\bmod (n+m, 2)=0$ we can partition the outer product expansion into $N$ products of linear combinations of the above and $N$ products of linear combinations of the complementary set. Alternately, if $\bmod (n+m, 2)=1$, then we have $2 N$ mixed products, each with one vector a combination of (45) or (46) and the companion a combination of the complementary set. 
But, while these annihilators appear to span the space containing the outer products, here again we lack an algorithm that generates the appropriate linear combinations. ${ }^{4}$ This is closely linked of course to the character of integrals in Section 3, there deduced by Gosper's general algorithm in lieu of an algebra specific to Jacobi polynomials. A first step in this direction is to make use of (45) and (46) with $j=\sigma=\tau=0$, from which it can be confirmed for $N=2 \ldots 5$ that

$$
\begin{aligned}
& \int_{-1}^{1} P_{m}^{(N-1 / 2, N-1 / 2)}(x) P_{n}^{(N-1 / 2, N-1 / 2)}(x) \frac{d x}{\sqrt{1-x^{2}}} \\
& =\frac{\Gamma(m+N+1 / 2) \Gamma(n+N+1 / 2)}{m \Gamma(m+2 N+1) \Gamma(n+1)} \sum_{k=1}^{N-1} \sum_{j=0}^{k}(-1)^{j+k} \\
& \times \frac{c_{k, j} \Gamma(m+k-j+2 N+1) \Gamma(n+j+1)}{\Gamma(m-k+j) \Gamma(n-j+2 N)}
\end{aligned}
$$

for certain $c_{k, j}>0$, all of which have an elementary small prime factorization. For $k=N-1$ we find

$$
c_{N-1, j}=\frac{2 \Gamma(N-j-1 / 2)}{\Gamma(N-j) \Gamma(j+1) \Gamma(2 N-j-1 / 2)},
$$

and we have therefore at last a partial analog of (14) in the statement

$$
\begin{aligned}
& \int_{-1}^{1} P_{m}^{(N-1 / 2, N-1 / 2)}(x) P_{n}^{(N-1 / 2, N-1 / 2)}(x) \frac{d x}{\sqrt{1-x^{2}}} \sim 2(-1)^{N-1} \\
& \times \frac{\Gamma(m+N+1 / 2) \Gamma(n+N+1 / 2) \Gamma(m+3 N) \Gamma(N-1 / 2)}{m \Gamma(m+1+2 N) \Gamma(m-N+1) \Gamma(2 N+n) \Gamma(N) \Gamma(-1 / 2+2 N)} \\
& \times{ }_{4} F_{3}\left(\begin{array}{c}
{[1-N, n+1,1-n-2 N, 3 / 2-2 N]} \\
{[1-m-3 N, 3 / 2-N, m-N+1]}
\end{array}\right),
\end{aligned}
$$

valid as the leading order approximation for large $m$ and $n$ (and exact for $N=$ 2). Evidently these results extend to all $N$. We hope to have a characterization of the remaining $c_{k, j}$ in the near future and a proof that (13) reduces to (47) for $M=N$ and $\alpha=\beta=-1 / 2$.

In the solution of (33) and (36), and the equivalent for $\mathbf{v}$, it will be clear that these linear equations partition into a homogeneous set independent of $\mu_{k}$ and the remainder, consisting in coefficients of $m$ from zero up to a degree $M$ given by the sum of the maximal degrees of $\mathbf{c}$ and $\mathbf{u}^{(0)}$. The consequence

\footnotetext{
${ }^{4}$ Also one needs to extend the forms above to general $(\alpha, \beta)$ but this is probably not difficult.
} 
of this structure is that the highest powers of $m$ in $\mathbf{c}$ are determined by a set of linear equations without reference to boundary conditions. We can use this fact in concert with the leading term in $m$ from each of the outer products, paralleling (36), to solve

$\sum_{j=0}^{n} \frac{\Gamma(m+2 j) \Gamma(m+2 j+2 N+1+k)}{\Gamma(m+2 j-k) \Gamma(m+2 j+2 N+1)} c_{2(N-j)+1}=0, \quad k=1, \ldots, N-1$,

as these must be satisfied by the full solution no matter what linear combinations of (45) arise. This system determines a polynomial solution of degree $N-1$ given by

$$
c_{2(N-j)+1}=c_{0}(-1)^{j} \frac{(m+2 j+N) \Gamma(m+3 N) \Gamma(m+j+N) \Gamma(N+1)}{\Gamma(j+1) \Gamma(N-j+1) \Gamma(m+j+2 N+1) \Gamma(m+N+1)} .
$$

Multiplying out these factors gives

$$
c_{2(N-j)+1}=c_{0}(-1)^{j}\left(\begin{array}{c}
N \\
j
\end{array}\right)\left[m^{N-1}+(N-1)(5 N / 2-j) m^{N-2}+\ldots\right]
$$

and from this $\Psi_{n} \sim P_{n}^{(-1 / 2,-1 / 2)}$ in the interior is shown to hold for arbitrary $N$ (see also the discussion in the next section). This solution has the much stronger property that the sum of all of its coefficients vanish, that is $\sum_{j=0}^{N} c_{2(N-j)+1}=0$, but only the first two remain unchanged by additional contributions from lower order terms appearing in the outer product. One may reasonably anticipate that this result can be extended to all the partial solutions, by using combinations of (45) and (46), and to arbitrary $(\alpha, \beta)$ by generalizing these expressions for the annihilators. That is to say, a proof of all the asymptotic aspects of these generalized orthogonal expansions for arbitrary $N$ appears within reach. But a proof that the solutions satisfy the expected families of boundary conditions seems to require that we find first the complete general outer product expansion and then the exact general solution for $\mathbf{c}$ as a function of the $\mu_{k}$, and this appears incomparably harder. (By construction, of course, such solutions would be auto-orthogonal.)

Sidestepping the issue of outer product representation, for $N=2$ we used (21) directly to verify that the polynomials satisfying $K_{1} f^{\prime}(1)+$ $f(1)=K_{2} f^{\prime}(-1)+f(-1)=0$ for $\alpha=\beta=-1 / 2$ are auto-orthogonal. We did the same for $N=3$ for the set satisfying $K_{1} f^{\prime \prime}(1)+K_{2} f^{\prime}(1)+f(1)=$ $K_{3} f^{\prime \prime}(-1)+K_{4} f^{\prime}(-1)+f(-1)=0$ again for $\alpha=\beta=-1 / 2$ and using $(22) .^{5}$ We omit a fuller discussion of $N=2,3$ paralleling that in Sections 4.1 and 4.2 as no new features emerge in the two-sided case.

\footnotetext{
${ }^{5}$ The details of these sets are given in [12].
} 


\section{The asymptotic properties of the basis sets}

So far we have only mentioned the asymptotic properties of some of the example basis sets in passing; here we expand on some of the general properties and, in particular, make the link to the "quasi- $L^{p}$ norm" part of the title. We start by remarking that expansions in the Jacobi polynomials themselves, $P_{n}^{(\alpha, \beta)}(x)$, have the property of exponential convergence, so the difference between an arbitrary function $f$ and its spectral representation is given by the asymptotic relation

$$
\left|f-\sum_{i=0}^{N} a_{n} P_{n}^{(\alpha, \beta)}(x)\right| \sim a_{N+1} P_{N+1}^{(\alpha, \beta)}(x), \quad N \rightarrow \infty .
$$

For special values of $\alpha=\beta$ the polynomials take on certain well-known optimal properties. For $\alpha=\beta=-1 / 2$ the right hand side is exactly equal ripple and thus the representation of $f$ in Chebyshev polynomials of the first kind minimizes the $L^{\infty}$ error for $N \rightarrow \infty$. Similar results hold for $\alpha=\beta=1 / 2$ and $\alpha=\beta=0$, which minimize the error in the $L^{1}$ and $L^{2}$ norms respectively. We conjecture that all basis sets $\Psi_{n}$ of Section 2 share the same asymptotic property, i.e., they minimize the $L^{p}$ error for the appropriate choices of $\alpha=\beta$. To show this requires that: (a) $\Psi_{n} \rightarrow P_{n}^{(\alpha, \beta)}$ (suitably normalized); and (b) the spectral coefficients in the expansion of any suitable test function (that is, one satisfying the same boundary conditions as the $\Psi_{n}$ ) converge to zero exponentially. Given the latter result, we can substitute $\Psi$ for $P$ in (51) and then appealing to (a), the minimization in the $L^{p}$ norm follows. We discuss these two properties in turn.

\subsection{The asymptotic form of $\Psi_{n}$}

In the simplest two-sided case where the boundary conditions impose that $f(1)=f^{\prime}(1)=\cdots=f^{(a-1)}(1)=f(-1)=f^{\prime}(-1)=\cdots=f^{(b-1)}(-1)=0$ for given integers $a, b$, the associated orthogonal basis set and asymptotic behavior is given by

$$
(1-x)^{a}(1+x)^{b} P_{n}^{(\alpha+2 a, \beta+2 b)} \sim c(-1)^{a} 2^{a+b} P_{n+a+b}^{(\alpha, \beta)}(x) .
$$

This result follows from [16, p. 194], Theorem 8.21.8 using the substitution $x=$ $\cos \theta$, thus demonstrating (a). For generalized boundary conditions we need a specific value of $N$ and for simplicity we use $N=2$. Additionally, we fix $\alpha=$ $\beta=-1 / 2$ although what follows generalizes easily to any $\alpha=\beta$. The following identity (easily proven by appeal to the Jacobi recurrence relation) is pivotal:

$$
P_{n}^{(3 / 2,3 / 2)}(x)=\frac{4 \Gamma(n+5 / 2)}{\sqrt{\pi} \Gamma(n+4)} \frac{x U_{n+1}(x)-(n+2) T_{n+2}(x)}{1-x^{2}}
$$


where $U_{n}$ and $T_{n}$ denote Chebyshev polynomials respectively of the first and second kinds. The most general boundary conditions for this set are

$$
\mu_{1} f^{\prime}(1)+\left(1-\mu_{1}\right) f(1)=\mu_{2} f^{\prime}(-1)+\left(1-\mu_{2}\right) f(-1)=0 .
$$

The associated auto-orthogonal basis for $n \geq 3$ is

$$
\Psi_{n}(x)=\sum_{i=1}^{5} c_{i} P_{n+2-i}^{(3 / 2,3 / 2)}(x),
$$

which is reasonably approximated in the interior by a two-term expansion of the $c_{i}$ in $n$. Normalizing the coefficients so that $c_{1} \sim 1$ as $n \rightarrow \infty$, the expansion assumes the form

$$
\begin{aligned}
\Psi_{n} \sim & \frac{4}{\sqrt{\pi}\left(1-x^{2}\right)} \\
& \times\left[\left(1+\frac{12}{n}\right)\left(x U_{n+2}(x)-(n+3) T_{n+3}(x)\right) \frac{\Gamma(n+7 / 2)}{\Gamma(n+5)}\right. \\
& -\left(2+\frac{30}{n}\right)\left(x U_{n}(x)-(n+1) T_{n+1}(x)\right) \frac{\Gamma(n+3 / 2)}{\Gamma(n+3)} \\
& \left.+\left(1+\frac{18}{n}\right)\left(x U_{n-2}(x)-(n-1) T_{n-1}(x)\right) \frac{\Gamma(n-1 / 2)}{\Gamma(n+5)}\right],
\end{aligned}
$$

and we remark that the boundary parameters $\mu_{1,2}$ do not appear until $\mathcal{O}\left(n^{-2}\right)$. Using the trigonometric form for the Chebyshev polynomials, it is easy to show that terms from the above, up to and including order $n^{-1}$, lead finally to

$$
\Psi_{n}(x) \sim \frac{16 n^{5} \Gamma(n-1 / 2)}{\sqrt{\pi} \Gamma(n+5)}\left[\left(1+\frac{23}{n}+\frac{16}{n^{2}}\right) T_{n+1}(x)+\left(\frac{7}{n}-\frac{16}{n^{2}}\right) x U_{n}(x)\right],
$$

in which form the leading order departure from equal ripple behavior in the interior is now explicit (thus (a) follows immediately) and this holds for all boundary conditions within this set. Note that the reduction to two Chebyshev polynomials free of the denominator of $\left(1-x^{2}\right)$ only occurs if the sum of the coefficients in (54) vanishes. This is true of the first two terms of the expansion in $n$-thus relying on a sum identity satisfied by the expansion coefficients beyond the leading binomial identity remarked earlier-but not thereafter, so higher order corrections lack a correspondingly simple expression.

Near $x= \pm 1, U_{n}$ approaches $( \pm 1)^{n}(n+1)$, so the second term is then of the same order as the first, consequently there is an order one departure from equal ripple, which manifests itself as a Gibbs-type overshoot (as illustrated in Fig. 1), which we do not analyze here. (This is easily done via local expansions about each of $x= \pm 1$ by means of the ${ }_{2} F_{1}$ representation of the Jacobi polynomials, which brings the boundary conditions in immediately. A 
typical result is an overshoot of 1.0863 at $x \approx 1-14.1055 / n^{2}$ for the basis set in Appendix B with $\alpha=\beta=-1 / 2$.) Intuitively, that the difference as $x \rightarrow \pm 1$ is $\mathcal{O}(1)$ is not surprising as $\Psi_{n}$ satisfies boundary conditions there whereas $P_{n+1}^{(\alpha, \beta)}$ (in this case $T_{n+1}$ ) does not. The nonuniform limit for $x \rightarrow \pm 1$ is the reason for terming these expansions quasi- $L^{p}$ norm.

Given $\mathbf{c}$, the above analysis can be extended using the appropriate generalization of (53) (similar forms for specific $N$ in the one-sided case are provided in the appendix). The key is that, as in (50), elements of $\mathbf{c}$ always satisfy

$$
c_{k+1} \sim(-1)^{k}\left(\begin{array}{l}
K \\
k
\end{array}\right), \quad k=0 \ldots K,
$$

where $K=N+1$ or $2 N+1$ for the one- or two-sided cases respectively, and with a similar form for terms of order $n^{-1}$. Property (a) then follows immediately for the interior since the same asymptotic relations hold for the expansion coefficients in the series that results after $N$ iterations of the index recurrence relation (66) for the one-sided case, or additionally with $N$ iterations of (67) in the two-sided case, to $P_{n}^{(\alpha, \beta)}(x)$.

\subsection{On exponential convergence}

The basic argument for exponential convergence is elementary. We outline it here using the "full" one-sided solution of Section 4.2, but again omit minor technical considerations in the interest of brevity. Additionally we do not attempt to find the sharpest classification of all functions for which the expansion coefficients decay faster than algebraically but rather for the sake of simplicity restrict the discussion to functions that have an absolutely convergent power series about $x=1$ with a radius of convergence $r_{c}>2$. While this excludes some admissible functions, the gain in economy of exposition is worth it.

Assume we seek the expansion of a function $f(x)$ that satisfies two arbitrary second-order boundary conditions of the form (37):

$$
f(x)=\sum_{n=1}^{\infty} f_{n} \hat{\Psi}_{n}^{(1)}(x),
$$

where we use the hat to denote a basis normalized according to (26). Using

$$
f_{n}=\int_{-1}^{1} f(x) \hat{\Psi}_{n}^{(1)} \frac{\mathrm{d} x}{\sqrt{1-x^{2}}}
$$

we now introduce

$$
f(x)=g(x)+\sum_{k=1}^{n-2} a_{k} \hat{\Psi}_{k}^{(1)}
$$


where

$$
\begin{aligned}
g(x) \equiv & \left(f(x)-\sum_{k=1}^{n-2} a_{k} \hat{\Psi}_{k}^{(1)}\right), \\
\mathbf{a} & =\left[\begin{array}{cccc}
\hat{\Psi}_{1} & \hat{\Psi}_{2} & \ldots & \hat{\Psi}_{n-2} \\
\hat{\Psi}_{1}^{\prime} & \hat{\Psi}_{2}^{\prime} & \ldots & \hat{\Psi}_{n-2}^{\prime} \\
\vdots & & & \\
\hat{\Psi}_{1}^{(n-1)} & \hat{\Psi}_{2}^{(n-1)} & \ldots & \hat{\Psi}_{n-2}^{(n-1)}
\end{array}\right]^{-1}\left[\begin{array}{c}
f(1) \\
f^{\prime}(1) \\
\ldots \\
f^{(n-1)}
\end{array}\right],
\end{aligned}
$$

(Matrix entries have their superscript label (1) suppressed to make room for the derivative order.) While the matrix in (58) is $n \times(n-2)$ and thus apparently overdetermined, because we consider only functions $f$ that satisfy the same boundary conditions, we are assured that the second expression in (58) in fact represents a consistent system with a unique solution.

Putting the second term in (57) in the integral in (56), the integral of the product with $\hat{\Psi}_{n}^{(1)}$ vanishes by auto-orthogonality. Now we observe that the first term in (57) has an expansion about $x=1$ of the form

$$
g=\sum_{k=n}^{\infty} \frac{f^{(k)}(1)}{k !}(x-1)^{k} .
$$

We interchange the order of summation and integration obtaining

$$
f_{n}=\sum_{k=n}^{\infty} \frac{f^{(k)}(1)}{k !} \int_{-1}^{1}(x-1)^{k} \hat{\Psi}_{n}^{(1)} \frac{\mathrm{d} x}{\sqrt{1-x^{2}}} .
$$

To evaluate the integral we use the expansion

$$
\hat{\Psi}_{n}^{(1)}(x) \sim \sqrt{\frac{2}{\pi}}\left[T_{n}(x)-\frac{7}{n}(1+x) U_{n-1}(x)\right]
$$

valid away from $x= \pm 1$ (see Section 5), derived from $\mathbf{c}_{1}$ and (72), and the integral formulae that

$$
\begin{aligned}
\int_{-1}^{1}(x-1)^{k} T_{n}(x) \frac{\mathrm{d} x}{\sqrt{1-x^{2}}} & =\frac{k}{n} \int_{-1}^{1}(x-1)^{k-1} U_{n-1}(x) \sqrt{1-x^{2}} \mathrm{~d} x \\
& =\frac{\pi}{2^{k}} \frac{(-1)^{n+k} \Gamma(2 k+1)}{\Gamma(k-n+1) \Gamma(k+n+1)} \\
& \sim(-1)^{n+k} 2^{k}\left[\sqrt{\frac{\pi}{k}}+\mathcal{O}\left(k^{-3 / 2}\right)\right]
\end{aligned}
$$

for $k \geq n$ and zero otherwise. If $\hat{\Psi}_{n}^{(1)}$ were replaced by $\hat{T}_{n}$ in (60), we would be computing the Chebyshev expansion coefficients. This is readily verified 
using $f(x)=1 /(x-3)$ and therefore $f^{(k)}(1) / k !=-1 / 2^{k+1}$, along with (62), which together then yield the correct (unnormalized) Chebyshev coefficients of $f_{n}=-(1+\sqrt{2})^{-2 n} / \sqrt{2}$.

The key point is that while (61) is inaccurate at leading order for $x \rightarrow 1$, the factor of $(x-1)^{k}$ in the integral turns this into an exponentially small error. Using (61) in (60) then incurs only an algebraically small relative error in the evaluation of $f_{n}$. From this two term approximation we have

$$
f_{n} \approx \sum_{k=n}^{\infty} \frac{f^{(k)}(1)}{k !}\left(1+\frac{7}{k}\right) \frac{\pi}{2^{k}} \frac{(-1)^{n+k} \Gamma(2 k+1)}{\Gamma(k-n+1) \Gamma(k+n+1)}
$$

and here we see that the difference between the (normalized) Chebyshev coefficients and those in the basis with $\Psi_{n}^{(1)}$ amounts in leading order to simply the correction factor of $7 / k$ in the sum. So exponential convergence of the latter expansion really derives from the same argument as that for coefficients of the Chebyshev expansion of the same function. That is, by our initial restriction of $f$, we know that $f^{(k)}(1) / k$ ! is bounded by a constant times $r_{c}^{-k}$ with $r_{c}>2$. Appealing to the asymptotic result in (62), the sum of the infinite series has a controlling factor of at $\operatorname{most} \exp \left(n \ln \left(2 / r_{c}\right)\right)$. (As one can see from the simple example above, this is generally too pessimistic an estimate, realized only for a function $f$ with a singularity on the real axis at $x=1-r_{c}$.)

Lastly we remark that we have not addressed completeness of the basis sets $\left\{\Psi_{n}^{(1)}\right\}_{n=1, \ldots, L}$. While they manifestly span the set of polynomials of any specified finite degree that satisfy the given boundary conditions, a proof that this continues to hold in the limit of infinite degree lies outside the scope of this paper.

\section{Extensions of auto-orthogonality}

\subsection{Orthogonality of polynomial derivatives}

Making the functions orthogonal, as we have done, is transparently helpful for expansions of many kinds. But this is not always the best way to approach solutions of a differential equation, for which the resulting matrices involve inner products of derivatives and may be full and poorly conditioned. Such matrices, being defined by a Sobolev-type inner product, may be rendered diagonal by using a modification of the canonical orthogonal polynomials (e.g. [14]). However, the imposition of boundary conditions remains unaddressed by the existing literature and we briefly discuss it here.

In [7] it is noted that an equation of order $2 k$ in which the function and its first $k-1$ derivatives vanish at the endpoints, the boundary conditions are automatically enforced by using

$$
\left(1-x^{2}\right)^{k} P_{n}^{(k, k)}(x)
$$


as the basis functions. This choice enjoys the further property that, owing to the identity

$$
\frac{d^{k}}{d x^{k}}\left(\left(1-x^{2}\right)^{k} P_{n}^{(k, k)}(x)\right)=(-2)^{k} \frac{\Gamma(n+1+k)}{\Gamma(n+1)} P_{n+k}^{(0,0)}(x),
$$

the "natural" projection of the equation leads to well-conditioned banded matrices of width $2(k-j)+1$ for derivative terms of order $2 j$, with $j=0 \ldots k$. Moreover the explicit matrix entries are relatively easy to find, in the form of rational polynomials in $(k, n)$.

An extension of this given in [8] for a fourth-order operator satisfying

$$
\begin{aligned}
y( \pm 1) & =0 \\
\beta y^{\prime}( \pm 1)+(1-\beta) y^{\prime \prime}( \pm 1) & =0,
\end{aligned}
$$

is to use

$$
\left(1-x^{2}\right)\left(1-\gamma_{j} x^{2}\right) P_{j}^{(1,1)}(x)
$$

where

$$
\gamma_{j}=\frac{(1-\beta)\left(j^{2}+3 j\right)+2}{(1-\beta)\left(j^{2}+3 j+8\right)+2} .
$$

While similar in spirit to the above, here the bandwidth is $2(k-j)+3$. Still, the matrices remain well-conditioned for solutions carried to degree 1800 (where the full problem involves two fields, one with the previous expansion and the other with this last form). Again the matrix elements can all be determined in closed rational form. (Useful details are given in Ierley and Worthing (unpublished manuscript) where the same expansions are used for a twodimensional problem and resulting sparse matrices up to $8000 \times 8000$ pose no roundoff problems.)

But, given the developments in this paper so far, it is natural to ask if our algorithm can be extended, so that we impose orthogonality between $(d / d x)^{k} \Psi_{n}(x)$ in place of that between $\Psi_{n}$, rendering the bandwidth of the derivative operators again $2(k-j)+1$ rather than $2(k-j)+3$. The short answer is yes, but seemingly only for the case $\alpha=\beta=0$. Additionally, as the problems above will suggest, we have in mind here instances where a differential equation of order $2 N$ can, via repeated integration by parts, result in projection matrices with derivatives up to order $N$ appearing symmetrically. This considerably restricts the class of admissible boundary conditions and also is the reason for requiring that $w(x)=1$. Ideally we might define orthogonality (for a fourth order problem) by

$$
\int_{-1}^{1} \Psi_{n}^{(4)} \Psi_{m} d x=0
$$

for $n \neq m$, rendering the projection of the highest derivative terms diagonal. However, for generalized boundary conditions this does not generate an 
auto-orthogonal set. In spite of these restrictions, applications for (partial) differential equations of this sort are common.

Consider a fourth order problem satisfying $y( \pm 1)=y^{\prime \prime}( \pm 1)=0$, where we attempt to construct a basis set whose second derivatives are orthogonal. According to Section 2.2 we require the sum of seven Jacobi polynomials for each basis function. However let us assume, like the boundary conditions, that the solutions have definite parity. Since $P_{n}^{(\alpha+N, \alpha+N)}(x)$ shares the same parity as $n$, the even solution may then be written

$$
\sum_{k=1}^{4} c_{k} P_{2(n+1-k)}^{(1,1)}(x),
$$

where we are required to use $y(1)=y^{\prime \prime}(1)=0$ along with a single orthogonality condition to determine the four unnormalized $c_{i}$. The construction above, using $N=1$ (rather than $N=3$ given in Section 2.2) for the $\Psi_{n}$, ensures that its second derivative is an equivalent sum with $N=3$ (using 68) and thus in a suitable form for auto-orthogonality. By extension, we may also ask for a basis set whose third derivatives are orthogonal by setting $N=0$, but it is clear that we can go no higher than this. The (unnormalized) first basis element is

$$
\left(1-x^{2}\right)\left(5-x^{2}\right),
$$

and the auto-orthogonal solutions are given by

$$
\begin{aligned}
& c_{1}=-(n+1)(n-1)(2 n-3)(4 n-7)(4 n-5), \\
& c_{2}=n\left(6 n^{2}-11 n+10\right)(4 n-7)(4 n-1), \\
& c_{3}=-(n-1)\left(6 n^{2}-7 n+7\right)(4 n-5)(4 n+1), \\
& c_{4}=n(n-2)(2 n-1)(4 n-1)(4 n+1) .
\end{aligned}
$$

In this instance it happens that the result can be reduced (modulo an overall scaling factor) to

$$
\begin{gathered}
n(4 n-1)(2 n-1) P_{2 n-4}(x)-(4 n-3)\left(4 n^{2}-6 n+5\right) P_{2 n-2}(x) \\
+(4 n-5)(n-1)(2 n-3) P_{2 n}(x) .
\end{gathered}
$$

While all of the material in the preceding sections can be exploited considerably more in this direction, we leave the matter here in the interest of brevity.

\subsection{Infinite domains}

On the Laguerre interval $[0, \infty]$ one also encounters auto-orthogonality, e.g. for the one-sided boundary condition $\psi_{n}(0)=0$, we have for $\psi_{1}=x$ and, appealing to the associated Laguerre polynomials to define

$$
\psi_{n}(x)=n L_{n}^{2}(x)-(n+2) L_{n-1}^{2}(x),
$$


that

$$
\int_{0}^{\infty} \psi_{n} \psi_{m} x \mathrm{e}^{-x} d x=\delta_{n, m}
$$

where the kernel is that for $L_{n}^{1}(x)$. Similar results extend to the general Laguerre kernel of argument $x^{k}$.

As with all the earlier examples in this paper, so too here it is immediate that the coefficients assume the limiting Pascal form (of $[1,-1])$ and it follows that the asymptotic limit of $\psi_{n}(x)$ as defined above is $n L_{n}^{1}(x)$ as expected. Moreover, this generalizes immediately to, e.g., a Robin boundary condition of

$$
(1-\mu) \psi_{n}(0)+\mu \psi_{n}^{\prime}(0)=0,
$$

with an associated three-term expansion of the form

$$
\psi_{n}(x)=\sum_{k=0}^{2} c_{k}(n ; \mu, \alpha) L_{n-k}^{2+\alpha}(x),
$$

for an orthogonality relation

$$
\int_{0}^{\infty} \psi_{n} \psi_{m} x^{\alpha} \mathrm{e}^{-x} d x=\delta_{n, m} .
$$

While for limitations of space we do not explore this any further, it is evident that the auto-orthogonality principle is equally applicable and hence, although our prior approach to the proof by means of annilator algebra might have appeared confined to the specific case of ${ }_{2} F_{1}$, it is perhaps not surprising it should apply to the confluent (Laguerre) case of ${ }_{1} F_{1}$. By the same token, one can reasonably anticipate that auto-orthogonality extends upward to the case of the "little q-Jacobi" polynomials

$$
p_{n}(x ; \alpha, \beta \mid q)={ }_{2} \phi_{1}\left(\begin{array}{c}
q^{-n}, \alpha \beta q^{n+1} \\
\alpha q
\end{array} \mid q ; q x\right)
$$

[10]. A necessary first step is establishing the equivalents of (14) and (48) in terms of ${ }_{4} \phi_{3}$.

\section{Discussion}

The results of this paper may be summarized in two observations. First, the Gram-Schmidt procedure with weight $(1-x)^{\alpha}(1+x)^{\beta}$ generates orthogonal polynomials with leading order asymptotic properties for the interior apparently independent of the linear homogenous boundary conditions imposed. Absent any boundary conditions, the conventional basis set $\left\{P_{n}^{(\alpha, \beta)}\right\}$ provides exponentially convergent spectral expansions. Based on the proof of this property for the one-sided solution in Section 5.2, we suggest that the Galerkin 
basis, $\left\{\Psi_{n}\right\}$, always exhibits exponential convergence in the quasi- $L^{p}$ norm for expansion of an arbitrary function satisfying the requisite boundary conditions, where $p=1,2, \infty$ when $\alpha=\beta$ take the values $1 / 2,0$, and $-1 / 2$ respectively. Second, the $\Psi_{n}$ may be written as a sum of a small number of Jacobi polynomials (the precise number dictated by the form of the boundary conditions) whose coefficients are determined by requiring orthogonality to only the first few basis functions, with auto-orthogonality following.

We have been able to prove auto-orthogonality and the admissible class of boundary conditions only for specific cases. But if one accepts the method provided in Section 2, given any $\alpha, \beta$, a basis set satisfying any linear homogeneous boundary conditions can be constructed. One can always check, a posteriori that the set is orthogonal and has the expected asymptotic properties. While the lack of a more general result thus makes little difference for practical application, yet there is apparently a deep algebraic structure associated with the weighted integrals of products of Jacobi polynomials that underlies the results in this paper, but whose most general expression has eluded us. In the one-sided case, we have a suitable form for the integral (14) and one requires only to relate this to the construction of the general outer product. From that result one should be able to prove (55) holds for the associated proper solution vector. For the two-sided problem we lack a complete equivalent to (14) beyond the leading order result provided in (48).

In a companion paper [11], we supply further numerical results on the use and implementation of Galerkin basis functions, with numerous specific cases of auto-orthogonal basis sets that satisfy a variety of physically motivated boundary conditions given in [12]. From a numerical perspective, there remains the issue of the accurate computation of $\Psi_{n}$, whose value particularly near $x \pm 1$ is effected, for instance in the two sided case, by a delicate cancellation between the $P_{n}^{(\alpha+N, \beta+N)}(x)$, which are individually $\mathcal{O}\left(n^{\alpha+N}\right)$. However, notwithstanding any better algorithm, the basis functions and any associated derivatives may be computed on any discrete set of grid points using computer algebra at high precision and saved to disk. This simply replaces the precomputation of basis functions in the numerical code itself that is required to perform "slow" matrix-vector multiplications, a necessity of the current lack of a fast (stable) Jacobi transform.

That we are the first (to our knowledge) to describe the construction and analysis of auto-orthogonal polynomials, given their obvious utility, is on the one hand unexpected. However, given the reliance of the proof on rather obscure non-canonical weighted integrals of products of Jacobi polynomials, it is perhaps not entirely surprising. Given this, the reader may wonder how we embarked upon this study. Our starting point was the construction of orthogonal functions with weight $\left(1-r^{2}\right)^{-1 / 2}$ on the domain $r \in[0,1]$ that satisfied boundary conditions only at $r=1$ and that were of the form $\Psi_{n}(r)=$ $r^{l} p\left(2 r^{2}-1\right)$ where $p$ is some polynomial and $l$ is a given positive integer. Such functions are relevant to the representation of functions in the radial variable $r$ both in cylindrical and spherical polar coordinate systems [3]. Such a basis set is obtained from the general one-sided construction with $x=2 r^{2}-1$ and 
$\alpha=-1 / 2, \beta=l-1 / 2$, and is probably the most obvious application of the one-sided case.

The issue of conditioning of these basis sets is of not only considerable theoretical interest, but also of practical significance. For a discretisation of, e.g., a second-order differential equation, of primary importance is the scaling (with $n$, the truncation) of the condition number of the matrix $A$ defined by the elements

$$
A_{i, j}=\int_{-1}^{1} \psi_{i}^{\prime \prime}(x) \psi_{j}(x)(1+x)^{\beta}(1-x)^{\alpha} d x
$$

For Galerkin basis sets that are orthogonal (as described in Section 2) this matrix is dense and, based on empirical tests, has a (2 norm) condition number that typically scales as $O\left(n^{4}\right)$. Though one finds the same for a naive Galerkin basis set of recombined Chebyshev polynomials, similar to the examples in Section 1, this simple comparison should not be taken as a definitive statement on relative accuracy of the two approaches; a simple model problem considered in [11] illustrates that the Jacobi basis proposed here may still yield significantly greater accuracy. Nonetheless, the generic scaling with $n^{4}$ does point to the desirability of other orthogonal formulations. Minimally, one would prefer where possible that the matrix $A$ be symmetric for optimal conditioning. While this is quite restrictive, it does include some very important practical cases, of particular note the diagonal form of the Laplacian in spherical coordinates, as discussed in detail in [11].

A more robust prospect of improved conditioning is some form of derivative orthogonality, as in Section 6, yielding not necessarily symmetric, but at least sparse, matrices of minimal bandwidth. A glimpse of what may be possible in that direction can be seen in [4], who show that, in the case of a biharmonic operator subject to $f( \pm 1)=f^{\prime}( \pm 1)=0$, a very remarkable advance is possible also using linear combinations of Jacobi polynomials (Eq. 3.4 in their paper). With the restriction $\alpha=\beta$ these polynomials are quasi-orthogonal where the matrix with elements defined by

$$
B_{i, j}=\int_{-1}^{1} \psi_{i}^{\prime}(x) \psi_{j}^{\prime}(x)(1+x)^{\alpha}(1-x)^{\alpha} d x
$$

is tridiagonal. This formulation leads to an amazing result that, by a judicious choice of preconditioner, the condition number for the problem remains $\mathcal{O}(N)$ and the complexity of the solver is $\mathcal{O}\left(N^{d+1}\right)$ in $d$ dimensions. How far generalization of this to what we might term "quasi-auto-orthogonality" for arbitrary boundary conditions and order may be possible is a fascinating prospect, but one necessarily the subject of future work.

Acknowledgements We wish to acknowledge postdoctoral support for PWL under NSF Grant 0724331. Also, a substantial portion of this work was done while GRI was on sabbatical leave as a guest of the Institute of Geophysics at the Eidgenössische Technische Hochschule in Zurich and he wishes to thank the Earth and Planetary Magnetism group there for their generous hospitality. 
Open Access This article is distributed under the terms of the Creative Commons Attribution Noncommercial License which permits any noncommercial use, distribution, and reproduction in any medium, provided the original author(s) and source are credited.

\section{Appendix A: Properties of Jacobi polynomials}

We make use of the following identities satisfied by Jacobi polynomials (see [13], p. 276)

$$
\begin{aligned}
(2 n+\alpha+\beta+2)(1-x) P_{n}^{(\alpha+1, \beta)} & =2(n+\alpha+1) P_{n}^{(\alpha, \beta)}-2(n+1) P_{n+1}^{(\alpha, \beta),} \\
(2 n+\alpha+\beta+2)(1+x) P_{n}^{(\alpha, \beta+1)} & =2(n+\beta+1) P_{n}^{(\alpha, \beta)}+2(n+1) P_{n+1}^{(\alpha, \beta)}, \\
(2 n+\alpha+\beta) P_{n}^{(\alpha-1, \beta)} & =(n+\alpha+\beta) P_{n}^{(\alpha, \beta)}-(n+\beta) P_{n-1}^{(\alpha, \beta)}, \\
(2 n+\alpha+\beta) P_{n}^{(\alpha, \beta-1)} & =(n+\alpha+\beta) P_{n}^{(\alpha, \beta)}+(n+\alpha) P_{n-1}^{(\alpha, \beta)}, \\
\frac{d^{k}}{d x^{k}} P_{n}^{(\alpha, \beta)}(x) & =\frac{\Gamma(\alpha+\beta+n+1+k)}{2^{k} \Gamma(\alpha+\beta+n+1)} P_{n-k}^{(\alpha+k, \beta+k)}(x) .
\end{aligned}
$$

For imposing boundary conditions, we make use of the following:

$$
\begin{aligned}
\left.\frac{d^{k}}{d x^{k}} P_{n}^{(\alpha, \beta)}(x)\right|_{x=1} & =\frac{2^{-k} \Gamma(n+k+\alpha+\beta+1) \Gamma(n+\alpha+1)}{\Gamma(n+\alpha+\beta+1) \Gamma(k+\alpha+1) \Gamma(n-k+1)}, \\
\left.\frac{d^{k}}{d x^{k}} P_{n}^{(\alpha, \beta)}(x)\right|_{x=-1} & =(-1)^{n-k} \frac{2^{-k} \Gamma(n+k+\alpha+\beta+1) \Gamma(n+\beta+1)}{\Gamma(n+\alpha+\beta+1) \Gamma(k+\beta+1) \Gamma(n-k+1)} .
\end{aligned}
$$

Lastly, the following identities are helpful in verifying the asymptotic equal ripple interior of the one-sided solutions for $\alpha=\beta=-1 / 2$

$$
\begin{aligned}
(1-x) P_{n}^{(3 / 2,-1 / 2)}(x)= & \frac{\Gamma(n+1 / 2)}{\sqrt{\pi} \Gamma(n+2)} \\
& \times\left[-2(n+1) T_{n+1}(x)+T_{n}(x)+x\left(U_{n}(x)+U_{n-1}(x)\right)\right]
\end{aligned}
$$

$$
\begin{aligned}
(1-x)^{2} P_{n}^{(5 / 2,-1 / 2)}(x)= & \frac{\Gamma(n+1 / 2)}{\sqrt{\pi} \Gamma(n+3)} \\
& \times\left[(2 n-1)(n+2) T_{n+2}(x)-(2 n+7)(n+1) T_{n+1}(x)\right. \\
& \left.+3 x\left(U_{n+1}(x)+U_{n}(x)\right)\right] .
\end{aligned}
$$




\section{Appendix B: A simple generalized basis set}

We present the full details of a basis set for $[-1,1]$ satisfying the boundary condition $\mu f^{\prime}(1)+(1-\mu) f(1)=0$. The first element of the basis set is the lowest order polynomial that can satisfy the boundary condition:

$$
\Psi_{1}=1+x(\mu-1)=-\frac{\mu(2+\alpha-\beta)-2 \alpha-6}{4+\alpha+\beta}+\frac{2(\mu-1)}{4+\alpha+\beta} P_{1}^{(\alpha+2, \beta)}(x) .
$$

By assuming $\Psi_{n}$ is of the form

$$
\Psi_{n}(x)=c_{1}(n) P_{n}^{(\alpha+2, \beta)}+c_{2}(n) P_{n-1}^{(\alpha+2, \beta)}+c_{3}(n) P_{n-2}^{(\alpha+2, \beta)}, \quad n \geq 2
$$

we may find $\left\{c_{1}, c_{2}, c_{3}\right\}$ up to a multiplicative constant using (15) to impose

$$
\int_{-1}^{1} \Psi_{1} \Psi_{n}(x)(1-x)^{\alpha}(1+x)^{\beta} d x=0
$$

and (69) to impose the boundary condition in $n$-space. The solution is

$$
\begin{aligned}
& c_{1}(n)=n(n+2+\alpha+\beta)(2 n+\beta+\alpha) p_{4}^{(1)}(n), \\
& c_{2}(n)=-(2 n+1+\beta+\alpha) p_{6}(n), \\
& c_{3}(n)=(n-1+\beta)(n+\alpha+1)(2 n+2+\beta+\alpha) p_{4}^{(2)}(n) .
\end{aligned}
$$

where $p_{i}(n)$ are polynomials in $n$ of degree $i$ given by

$$
\begin{aligned}
p_{4}^{(1)}(n)= & (\alpha+1)\left(9 \mu^{2} \alpha^{2}+4 \alpha^{2}-12 \alpha^{2} \mu-56 \mu \alpha+6 \mu^{2} \alpha \beta+39 \mu^{2} \alpha-4 \mu \beta \alpha\right. \\
& \left.+20 \alpha+24-12 \mu \beta+38 \mu^{2}-60 \mu+15 \mu^{2} \beta+\mu^{2} \beta^{2}\right)-n \mu(\alpha+\beta) \\
& \times\left(-4 \alpha^{2}+6 \alpha^{2} \mu+2 \mu \beta \alpha+22 \mu \alpha-16 \alpha-12+16 \mu+3 \mu \beta\right) \\
& +n^{2} \mu\left(-3 \mu \beta+2 \mu \beta \alpha-22 \mu \alpha+2 \alpha^{2} \mu \beta+\alpha^{3} \mu+2 \mu \beta^{2}-16 \mu\right. \\
& \left.-4 \alpha^{2} \mu+16 \alpha+\mu \beta^{2} \alpha+4 \alpha^{2}+12\right) \\
& +2 n^{3} \mu^{2}(\alpha+2)(\alpha+\beta)+n^{4} \mu^{2}(\alpha+2) \\
p_{6}(n)= & (-1+\mu)(\alpha+3)(\alpha+2)(\alpha+1)(2+\alpha+\beta) \\
& \times(3 \mu \alpha-2 \alpha+\mu \beta+2 \mu-2) \\
& -n(\alpha+\beta+1)\left(5 \mu^{2} \alpha^{4}-4 \alpha^{4} \mu-8 \alpha^{3} \mu-4 \alpha^{3} \mu \beta+23 \mu^{2} \alpha^{3}\right. \\
& +6 \mu^{2} \alpha^{3} \beta-8 \alpha^{3}+\mu^{2} \beta^{2} \alpha^{2}+26 \mu^{2} \alpha^{2} \beta-48 \alpha^{2}-16 \alpha^{2} \mu \beta \\
& +12 \mu^{2} \alpha^{2}+52 \alpha^{2} \mu+3 \mu^{2} \beta^{2} \alpha-88 \alpha-52 \mu^{2} \alpha+152 \mu \alpha-12 \mu \beta \alpha \\
& \left.+28 \mu^{2} \alpha \beta+96 \mu-48-48 \mu^{2}+4 \mu^{2} \beta^{2}+8 \mu^{2} \beta\right)
\end{aligned}
$$




$$
\begin{aligned}
& +n^{2}\left(48-66 \mu^{2} \alpha^{3}+88 \alpha-72 \mu-72 \mu \alpha+48 \mu \beta+124 \mu \beta \alpha\right. \\
+ & 32 \mu \beta^{2} \alpha-46 \mu^{2} \alpha-8 \mu^{2} \alpha^{4}+\mu^{2} \beta^{3} \alpha+48 \alpha^{2} \\
& -11 \mu^{2} \alpha^{3} \beta+\mu^{2} \beta^{3} \alpha^{2}-72 \mu^{2} \beta+12 \alpha^{4} \mu-36 \mu^{2} \beta^{2}+\mu^{2} \alpha^{5} \\
& +20 \alpha^{3} \mu \beta+3 \mu^{2} \beta^{2} \alpha^{3}+56 \alpha^{3} \mu+96 \alpha^{2} \mu \beta-35 \mu^{2} \beta^{2} \alpha \\
& -2 \mu^{2} \beta^{2} \alpha^{2}+44 \alpha^{2} \mu+16 \mu^{2}-117 \mu^{2} \alpha^{2}+3 \mu^{2} \alpha^{4} \beta+24 \mu \beta^{2} \\
& \left.-101 \mu^{2} \alpha^{2} \beta-159 \mu^{2} \alpha \beta+8 \alpha^{3}+8 \mu \beta^{2} \alpha^{2}\right) \\
& +2 n^{3} \mu(\alpha+\beta+1)\left(2 \alpha^{3} \mu+8 \alpha^{2}-\alpha^{2} \mu+3 \alpha^{2} \mu \beta+7 \mu \beta \alpha\right. \\
& \left.-29 \mu \alpha+32 \alpha+\mu \beta^{2} \alpha-30 \mu+2 \mu \beta^{2}+4 \mu \beta+24\right) \\
& +n^{4} \mu\left(13 \alpha^{2} \mu \beta+24+37 \mu \beta \alpha+7 \alpha^{3} \mu+8 \alpha^{2}+6 \mu \beta^{2} \alpha+32 \alpha\right. \\
& \left.+12 \mu \beta^{2}-4 \mu \alpha+19 \alpha^{2} \mu-20 \mu+24 \mu \beta\right) \\
& +6 n^{5} \mu^{2}(\alpha+2)(\alpha+\beta+1)+2 n^{6} \mu^{2}(\alpha+2) \\
& (-1+\mu)^{2}(\alpha+3)(\alpha+2)(\alpha+1)-n \mu(2+\alpha+\beta) \\
& \times\left(4 \alpha^{2} \mu-4 \alpha^{2}-16 \alpha+16 \mu \alpha-\mu \beta-12+12 \mu\right) \\
& +n^{2} \mu\left(12+\mu \beta^{2} \alpha+2 \alpha^{2} \mu \beta+8 \mu \beta \alpha+4 \alpha^{2}+2 \mu \beta^{2}+16 \alpha\right. \\
& \left.-4 \mu \alpha+2 \alpha^{2} \mu-4 \mu+\alpha^{3} \mu+9 \mu \beta\right) \\
& +2 n^{3} \mu^{2}(\alpha+2)(2+\alpha+\beta)+n^{4} \mu^{2}(\alpha+2) \\
p_{4}^{(2)}(n)= & \\
&
\end{aligned}
$$

Orthogonality can be proven by making use of (15) assisted by computer algebra. Note also that the $c_{i}$ take on the ratio $[1,-2,1]$ as $n \rightarrow \infty$. In the particular case of $\mu=0$, both $p_{4}^{(1)}$ and $p_{4}^{(2)}$ reduce to a constant and $p_{6}$ reduces to a polynomial of degree two. When $\mu=1$, each $c_{i}$ has a common factor of $n(n+2+\alpha+\beta)$, which can be removed, leaving the resulting expressions as polynomials of degree five.

\section{References}

1. Askey, R.: Orthogonal Polynomials and Special Functions. SIAM, Philadelphia (1975)

2. Andrews, G.E., Burge, W.H.: Determinant identities. Pac. J. Math. 158, 1-14 (1993)

3. Boyd, J.P.: Chebyshev and Fourier Spectral Methods. Dover, New York (2001)

4. Doha, E.H., Bhrawy, A.H.: A Jacobi spectral Galerkin method for the integrated forms of fourth-order elliptic differential equations. Numer. Methods Partial Differ. Equ. 25(3) (2009)

5. Golub, G.H., Van Loan, C.F.: Matrix Computations, third edn. Johns Hopkins, Baltimore (1996)

6. Gosper, R.W., Jr.: Decision procedure for indefinite hypergeometric summation. Proc. Natl. Acad. Sci. USA 75, 40-42 (1977)

7. Ierley, G.R.: A class of sparse spectral operators for inversion of powers of the Laplacian in $\mathrm{N}$ dimensions. J. Sci. Comp. 12, 57-73 (1997)

8. Ierley, G.R., Kerswell, R.R., Plasting, S.C.: Infinite Prandtl number convection. Part 2: a singular limit of upper bound theory. J. Fluid Mech. 560, 159-227 (2005) 
9. Ismail, M.: Classical and quantum orthogonal polynomials in one variable. In: Encyclopedia of Mathematics and its Applications, vol. 98. CUP (2005)

10. Koekoek, R., Swarttouw, R.F.: The Askey-scheme of hypergeometric orthogonal polynomials and its q-analogue. Delft University of Technology, Faculty of Information Technology and Systems, Department of Technical Mathematics and Informatics, Report no. 98-17 (1998)

11. Livermore, P.: Galerkin orthogonal polynomials. J. Comp. Phys. (2009, in press)

12. Livermore, P.: A compendium of Galerkin orthogonal polynomials. Tech. Rep., Scripps Institution of Oceanography, UC San Diego. Available at http://escholarship.org/uc/item/ $9 \mathrm{vk} 1 \mathrm{c} 6 \mathrm{~cm}$

13. Luke, Y.: The Special Functions and Their Approximations, vol. 1, first edn. Academic, London (1969)

14. Marcellan, F., Ronveaux, A.: On a class of polynomials orthogonal with respect to a discrete Sobolev inner product. Indag. Math., N.S. 1(4), 451-464 (1990)

15. Mason, J.C., Handscomb, D.C.: Chebyshev Polynomials. Chapman \& Hall, London (2003)

16. Szegö, G.: Orthogonal Polynomials, fourth edn. American Mathematical Society, Providence (1975)

17. Weber, M., Erdelyi, A.: On the finite difference analogue of Rodrigues' formula. Am. Math. Mon. 59, 163-168 (1952) 